\title{
DIRAC: A High Resolution Spectrometer for Pionium Detection
}

B. Adeva ${ }^{\mathrm{p}, *}$, L. Afanasyev $^{\ell}, \mathrm{M}$. Benayoun ${ }^{\mathrm{e}}$, A. Benelli $^{\mathrm{q}}$,

Z. Berka ${ }^{\mathrm{b}}$, V. Brekhovskikh ${ }^{\circ}$, G. Caragheorgheopol ${ }^{\mathrm{m}}$, T. Cechak ${ }^{\mathrm{b}}$, M. Chiba $^{\mathrm{k}}$, E. Cima ${ }^{\mathrm{f}}$, S. Constantinescu ${ }^{\mathrm{m}}$, C. Detraz ${ }^{a}$, D. Dreossi ${ }^{\mathrm{g}}$, D. Drijard ${ }^{\mathrm{a}}$, A. Dudarev ${ }^{\ell}$,

I. Evangelou d, M. Ferro-Luzzi ${ }^{\text {a }}$, M.V. Gallas ${ }^{\text {pa }}$, J. Gerndt ${ }^{\text {b }}$, R. Giacomich ${ }^{\mathrm{g}}$, P. Gianotti ${ }^{\mathrm{f}}$, M. Giardoni ${ }^{\mathrm{f}}$, D. Goldin ${ }^{\mathrm{q}}$, F. Gómez ${ }^{\mathrm{p}}$, A. Gorin ${ }^{\circ}$, O. Gortchakov ${ }^{\ell}$, C. Guaraldo ${ }^{\mathrm{f}}$, M. Hansroul ${ }^{\text {a }}$, M. Iliescu ${ }^{\text {f,m }}$, M. Zhabitsky ${ }^{\ell}$, V. Karpukhin ${ }^{\ell}$, J. Kluson $^{\text {b }}$, M. Kobayashi ${ }^{\text {h, P. Kokkas }}{ }^{\text {, }}$, V. Komarov ${ }^{\ell}$, V. Kruglov ${ }^{\ell}$, L. Kruglova ${ }^{\ell}$, A. Kulikov ${ }^{\ell}$, A. Kuptsov ${ }^{\ell}$, V. Kurochkin ${ }^{\circ}$, K.-I. Kuroda ${ }^{\ell}$, A.Lamberto ${ }^{\mathrm{g}}$, A. Lanaro ${ }^{\mathrm{a}, \mathrm{f}}$, V. Lapshin ${ }^{\circ}$, R. Lednicky ${ }^{c}$, P. Leruste ${ }^{\mathrm{e}}$, P. Levisandri ${ }^{\mathrm{f}}$, A. Lopez Aguera ${ }^{p}$, V. Lucherini ${ }^{f}$, T. Maki ${ }^{j}$, N. Manthos ${ }^{d}$, I. Manuilov ${ }^{\circ}$, L. Montanet $^{\mathrm{a}}$, J.-L. Narjoux ${ }^{\mathrm{e}}$, L. Nemenov ${ }^{\mathrm{a}, \ell}$,

M. Nikitin ${ }^{\ell}$, T. Núñez Pardo ${ }^{p}$, K. Okada $^{\mathrm{i}}$, V. Olchevskii ${ }^{\ell}$,

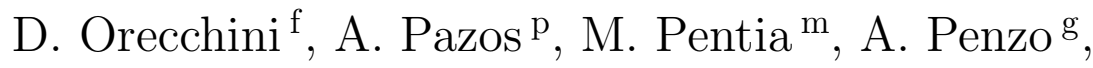
J.-M. Perreau ${ }^{\mathrm{a}}$, C. Petrascu ${ }^{\mathrm{f}, \mathrm{m}}$, M. Pló $^{\mathrm{p}}$, T. Ponta $^{\mathrm{m}}$, D. Pop ${ }^{\mathrm{m}}$, G.F.Rappazzo ${ }^{\mathrm{g}}$, A. Riazantsev ${ }^{\mathrm{o}}$, J.M. Rodriguez ${ }^{\mathrm{p}}$,

A. Rodriguez Fernandez ${ }^{\mathrm{p}}$, A. Romero ${ }^{\mathrm{p}}$, V. Rykalin ${ }^{\mathrm{o}}$, C. Santamarina ${ }^{p, q}$, J. Saborido ${ }^{p}$, J. Schacher ${ }^{r}$, Ch.P. Schuetz ${ }^{q}$, A. Sidorov ${ }^{\text {}}$, J. Smolik ${ }^{c}$, M. Steinacher ${ }^{q}$, F. Takeutchi ${ }^{i}$, A. Tarasov $^{\ell}$, L. Tauscher ${ }^{\mathrm{q}}$, M.J. Tobar ${ }^{\mathrm{p}}$, F. Triantis ${ }^{\mathrm{d}}$, S. Trusov ${ }^{n}$, V. Utkin $^{\ell}$, O. Vázquez Doce ${ }^{\mathrm{p}}, \mathrm{P}$. Vázquez $^{\mathrm{p}}$, S. Vlachos ${ }^{\mathrm{q}}$, V. Yazkov ${ }^{\mathrm{n}}$, Y. Yoshimura ${ }^{\mathrm{h}}, \mathrm{P}$. Zrelov ${ }^{\ell}$

${ }^{a}$ CERN, Geneva, Switzerland

${ }^{\mathrm{b}}$ Czech Technical University, Prague, Czech Republic

${ }^{\mathrm{c}}$ Institute of Physics ACSR, Prague, Czech Republic

${ }^{\mathrm{d}}$ Ioannina University, Ioannina, Greece

${ }^{\mathrm{e}}$ LPNHE des Universites Paris VI/VII, IN2P3-CNRS, France

${ }^{\mathrm{f}}$ INFN - Laboratori Nazionali di Frascati, Frascati, Italy 
${ }^{\mathrm{g}}$ INFN-Trieste and Trieste University, Trieste, Italy

${ }^{\mathrm{h}}$ KEK, Tsukuba, Japan

${ }^{\mathrm{i}}$ Kyoto Sangyou University, Japan

${ }^{\mathrm{j}}$ UOEH-Kyushu, Japan

k Tokyo Metropolitan University, Japan

${ }^{\ell}$ JINR Dubna, Russia

${ }^{\mathrm{m}}$ National Institute for Physics and Nuclear Engineering IFIN-HH Bucharest, Romania

${ }^{\mathrm{n}}$ Skobeltsin Institute for Nuclear Physics of Moscow State University Moscow, Russia

${ }^{\circ}$ IHEP Protvino, Russia

p Santiago de Compostela University, Spain

${ }^{\mathrm{q}}$ Basel University, Switzerland

${ }^{\mathrm{r}}$ Bern University, Switzerland

\begin{abstract}
The DIRAC spectrometer has been commissioned at CERN with the aim of detecting $\pi^{+} \pi^{-}$atoms produced by a $24 \mathrm{GeV} / c$ high intensity proton beam in thin foil targets. A challenging apparatus is required to cope with the high interaction rates involved, the triggering of pion pairs with very low relative momentum, and the measurement of the latter with resolution around $0.6 \mathrm{MeV} / c$. The general characteristics of the apparatus are explained and each part is described in some detail. The main features of the trigger system, data-acquisition, monitoring and setup performances are also given.
\end{abstract}

Key words: DIRAC experiment, double arm spectrometer, pion scattering, experimental techniques, elementary atom

\footnotetext{
* Departamento de Física de Partículas, Universidade de Santiago de Compostela, E-15782 Santiago, Spain. Tel.: 34-981-563100, e-mail: adevab@usc.es
} 


\section{Introduction}

The DIRAC experiment aims to measure the ground state lifetime of $\pi^{+} \pi^{-}$ atoms with $10 \%$ precision, using the $24 \mathrm{GeV} / c$ proton beam of the CERN Proton Synchrotron. The atom lifetime is a consequence of the strong interaction at low energy and it is determined by the charge exchange amplitude $\pi^{+} \pi^{-} \rightarrow \pi^{0} \pi^{0}$ very close to threshold. The probability of this process is proportional to the square of the difference of S-wave $\pi \pi$ scattering lengths with isotopic spin 0 and $2,\left|a_{0}-a_{2}\right|^{2}$. The relation between the lifetime and $\left|a_{0}-a_{2}\right|$ is model-independent [1]. The pion scattering lengths have been calculated in the framework of chiral perturbation theory with a precision of a few percent: $a_{0}=0.220 \pm 0.005$ and $a_{2}=-0.0444 \pm 0.0010$. Using these values one can predict the pionium lifetime [2]: $(2.9 \pm 0.1) \times 10^{-15} s$. In order to determine $\left|a_{0}-a_{2}\right|$ down to $5 \%$, the lifetime has to be measured within $10 \%$ accuracy. Such a measurement would provide a crucial test for the understanding of chiral symmetry breaking in QCD.

Pionium atoms $\left(A_{2 \pi}\right)$ are produced in proton-nucleus interactions. After production these relativistic atoms may either decay into $\pi^{0} \pi^{0}$ or get excited to higher quantum numbers, or break up (be ionised) in the target material where they are produced. In the case of break-up, characteristic pion pairs ("atomic" pairs) emerge. These pairs have a low relative momentum in their centre of mass system $(Q<3 \mathrm{MeV} / c)$, very small opening angle $(\theta<3 \mathrm{mrad})$ and nearly identical energies in the laboratory system. A high resolution magnetic spectrometer is then required [3] to split up the pairs and measure their relative momentum with sufficient precision $(0.6 \mathrm{MeV} / c)$ to detect the pionium signal superimposed on the substantial background of "free" $\pi^{+} \pi^{-}$pairs pro-

duced in inclusive proton-nucleus interactions. A previous experiment, using internal proton beam, has reported observation of pionium atoms [4].

The total number of produced $\pi^{+} \pi^{-}$atoms is related by an exact expression to the number of free pion pairs with low relative momenta. For a given target material and thickness the ratio of observed atomic pairs to the total number of produced atoms, i.e. the atom breakup probability, depends on the lifetime in a unique way [5].

\section{General layout of the experimental setup}

The DIRAC experimental setup $[6,7]$ is located at the T8 proton beam line of $24 \mathrm{GeV} / \mathrm{c}$ momentum in the East Hall of the PS accelerator at CERN. The isometric view of the setup is shown in Fig.1. The DIRAC apparatus 
is designed to detect charged pion pairs with high resolution over the pair relative momentum. It became operational at the end of 1998 and has been collecting data since the middle of 1999.

Fig. 1. Isometric view of the DIRAC setup. The radiation shielding boundaries are shown on the floor (each division marked on the boundary corresponds to 1 meter).

Fig. 2. Side view of the DIRAC setup. The secondary particle channel is inclined by $5.7^{\circ}$ with respect to the primary proton beam.

The setup consists of the proton beam line, target station, secondary particle vacuum channel, spectrometer magnet and detectors placed upstream and downstream the analysing magnet. Free and atomic $\pi^{+} \pi^{-}$pairs produced in the target enter the secondary particle channel which is tilted upwards by $5.7^{\circ}$ with respect to the proton beam (Fig.2). At the end of the secondary particle channel the spectrometer magnet is installed, also tilted by $5.7^{\circ}$ together with all the downstream detectors.

The top view of the setup is shown in Fig.3. The upstream section of the secondary particle channel between the target station and the spectrometer magnet is instrumented with the following detectors: microstrip gas chambers (GEM/MSGC), scintillating fibre detector (SFD) and scintillation ionisation hodoscope (IH).

Downstream the spectrometer magnet the setup splits into two identical arms for detection and identification of positive and negative charged particles. The angle between each arm and the spectrometer symmetry axis is $19^{\circ}$. Along each arm the following detectors are located: drift chamber system (DC), vertical scintillation hodoscope $(\mathrm{VH})$, horizontal scintillation hodoscope $(\mathrm{HH})$, gas Cherenkov counter $(\mathrm{CH})$, preshower detector (PSH) and muon detector (MU).

\section{Beam lines, spectrometer magnet and radi- ation shielding}

\subsection{Proton beam and target station}

To extract protons from the PS to the T8 beam line a slow ejection mode is used. The beam is extracted in spills of $\approx 400-500 \mathrm{~ms}$ duration. During data taking, between 1 to 5 cycles per PS super-cycle of $14.4 \div 19.2 \mathrm{~s}$ duration are 


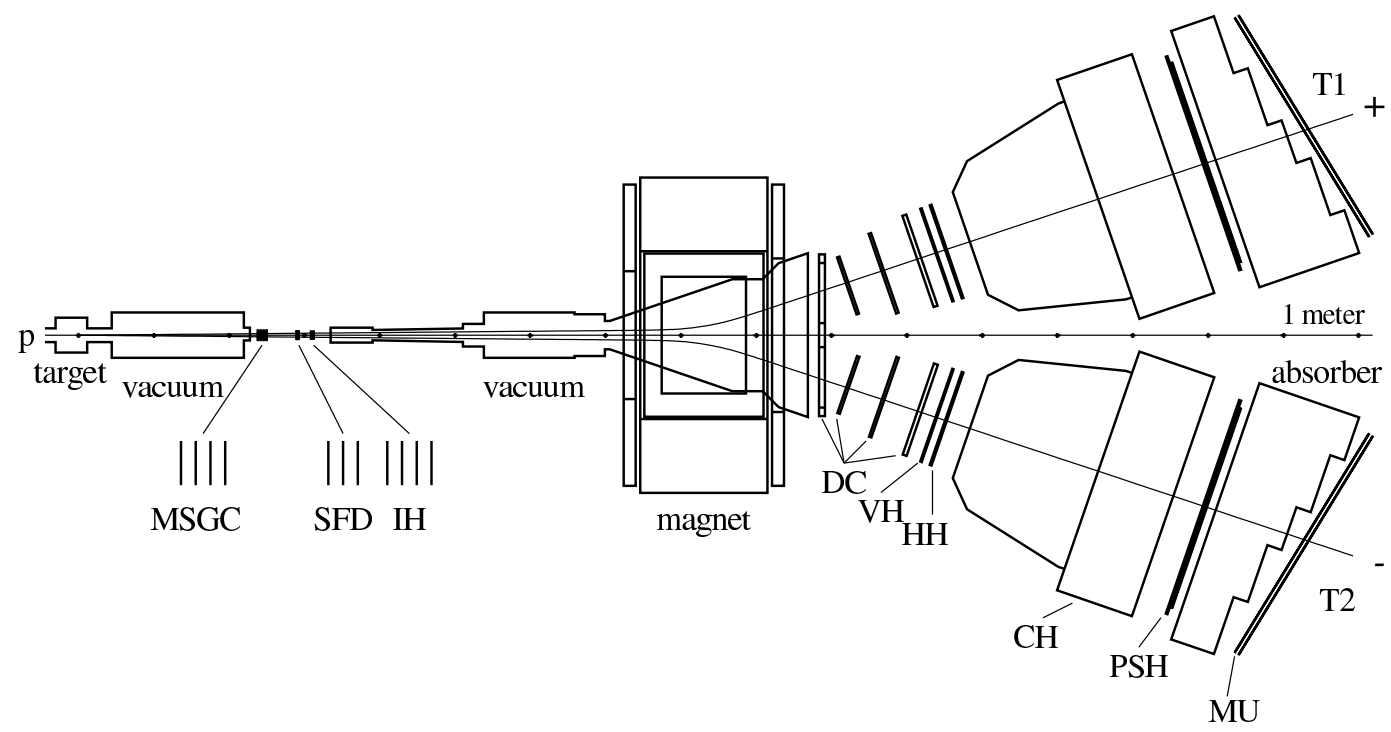

Fig. 3. Schematic top view of the DIRAC spectrometer. Moving from the target station towards the magnet there are 4 planes of microstrip gas chambers (GEM/MSGC), 3 planes of scintillating fibre detectors (SFD) and 4 planes of ionisation hodoscope (IH). Downstream the dipole magnet, on each arm of the spectrometer, there are 4 stations of drift chambers (DC), vertical and horizontal scintillation hodoscopes $(\mathrm{VH}, \mathrm{HH})$, gas Cherenkov counter $(\mathrm{CH})$, preshower detector $(\mathrm{PSH})$ and, behind the iron absorber, muon detector (MU).

delivered to DIRAC. The proton beam intensity was set to $(0.6-1.0) \cdot 10^{11}$ protons per spill, depending on the target used.

The PS proton beam line includes two bending magnets deflecting the beam at an angle of $76 \mathrm{mrad}$ towards the final straight T8 section, corrector magnets performing horizontal and vertical steering and quadrupole magnets which focus the beam on the experiment target. The dimension of the beam spot at the target location are $x=1.6 \mathrm{~mm}, y=3.2 \mathrm{~mm}$ at $2 \sigma$ level. The divergence of the beam is about $1 \mathrm{mrad}$. The nominal momentum of the extracted beam is $24 \mathrm{GeV} / c$, with instantaneous momentum spread close to $0.08 \%$ at $2 \sigma$. The design of the proton beam line optics has been optimised using the TRANSPORT simulation code [8] by the CERN PS Division.

Downstream the target the proton beam travels in a vacuum channel below the spectrometer magnet and detectors and finally is absorbed by a beam dump.

To measure the beam intensity and to tune the beam position on the target, the beam line is equipped at several locations with secondary emission chambers and luminescent screens with TV cameras. One additional beam position detector (centroid) [9] is installed close to the target station. 
The target station houses a remote controlled device with 12 holders for the targets, including an empty holder and a luminescence screen. During data taking, targets made of Pt $(28 \mu \mathrm{m}$ thick $), \mathrm{Ni}(94 \mu \mathrm{m}$ and $98 \mu \mathrm{m}$ thick $)$ and Ti $(250 \mu \mathrm{m}$ thick $)$ were used.

The DIRAC experiment is sensitive to particles outside the beam core (halo), because the target is very thin (nuclear target efficiency is $<10^{-3}$ ), and the upstream detectors are placed very close (18-26 cm in the vertical direction) to the primary proton beam. The halo is originated from scattering of primary protons on the splitter blades, and a special optics has been designed to decrease the background halo to a negligible level. The ratio of detector counting rates with the target in place to those with an empty holder was measured to be $\sim 25$.

\subsection{Secondary particle channel and spectrom- eter magnet}

The secondary particle channel [6] [7] is placed at an angle of $5.7^{\circ}$ relative to the proton beam and consists of two vacuum volumes, as shown in Fig. 3. The first one is a $2 \mathrm{~m}$ long, $611 \mathrm{~mm}$ diameter tube, located immediately downstream the target station, common to both the proton beam line and the secondary particle channel. Secondary particles exit this tube through a $200 \mathrm{~mm}$ diameter window, made of $250 \mu \mathrm{m}$ thick mylar film. The second volume, located at $\sim 3.5 \mathrm{~m}$ from the target, consists of a cylindrical vacuum section, containing a collimator, attached to a $2.7 \mathrm{~m}$ long flat vacuum chamber placed between the spectrometer magnet poles. A $1.5 \mathrm{~m}$ long air gap between the two vacuum sections allows insertion of the upstream detectors. The angular aperture of the secondary particle channel is determined by the collimator and is equal to $\pm 1^{\circ}$ in horizontal and vertical directions resulting in a solid angle acceptance of $1.2 \cdot 10^{-3} \mathrm{sr}$. The flat chamber is ended with a $0.68 \mathrm{~mm}$ thick $\mathrm{Al}$ outlet window of $2.0 \times 0.4 \mathrm{~m}^{2}$ dimensions $(W \times H)$.

The spectrometer dipole magnet (magnetic field $\mathrm{B}=1.65 \mathrm{~T}$, field integral $\mathrm{BL}=$ $2.2 \mathrm{~T} \cdot \mathrm{m})$ has an aperture of $1.55 \times 0.50 \mathrm{~m}^{2}(W \times H)$. To reduce the stray field, two magnetic screens are fixed near its entrance and exit.

Table 1 summarises the material thicknesses (in units of radiation length $\times 10^{-4}$ ) encountered by secondary particles before they reach the DC system where their momenta are measured. 
Table 1

Material contributions along the secondary particle channel in units of radiation length $\times 10^{-4}$.

\begin{tabular}{|l|r|}
\hline Ni-target & 33.5 \\
\hline Mylar window & 8.7 \\
4 planes GEM/MSGC & 224.1 \\
3 planes SFD & 260.0 \\
4 planes IH & 153.1 \\
air gap & 34.7 \\
Mylar window & 8.7 \\
Al-window & 76.4 \\
Total & 765.7 \\
\hline
\end{tabular}

\subsection{Beam dump and radiation shielding}

Neutron and gamma fluxes may cause a serious problem to sensitive elements of the setup. To estimate their effect a simulation of the background radiation flux in the full experimental apparatus has been performed [10]. The results were used to optimise the design of the radiation shielding which is shown in Fig. 4.

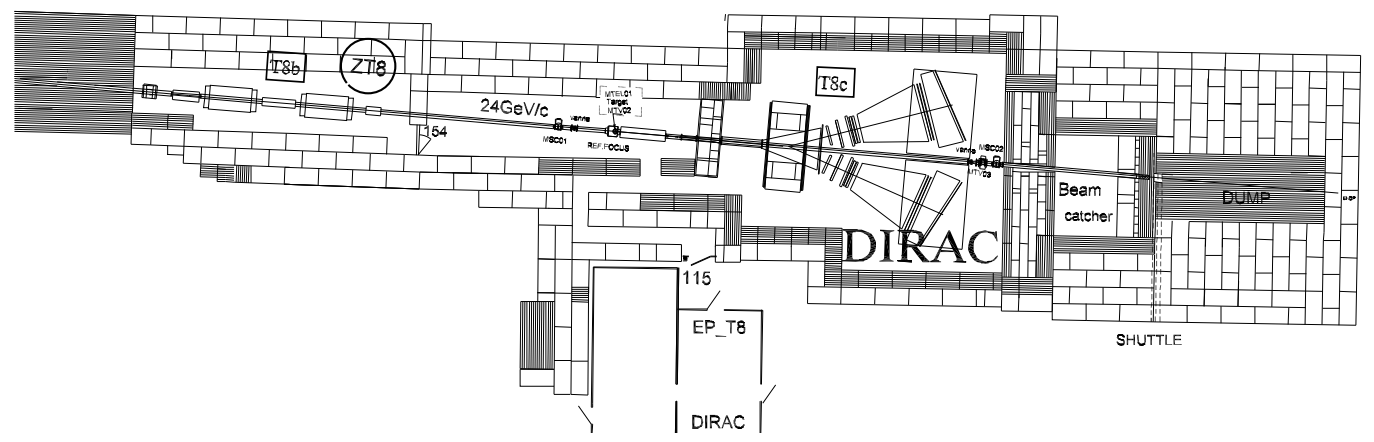

Fig. 4. The DIRAC setup on the T8 PS extraction line and the radiation shielding.

At the end of the T8 beam line primary protons are absorbed by an iron beam dump. To decrease the background gamma and neutron fluxes from the beam dump towards the detectors, a dedicated radiation shielding has been adopted. It includes installation of a graphite core into the beam dump area, a concrete wall near the beam dump and, at $3 \mathrm{~m}$ distance, another iron-concrete-iron wall, both with holes to allow passage of the proton beam pipe.

The downstream detectors are shielded in addition from background secondary particles produced on the primary proton pipe and surrounding elements. For this purpose a $1 \mathrm{~m}$ thick iron wall is installed between the upstream detector 
region and the spectrometer magnet. In addition, collimators are inserted both in the primary proton beam pipe and in the secondary particle channel. The presence of the collimator in the proton beam line determines a reduction of the background rate by a factor of 2 .

A radiation shielding encloses the whole DIRAC experimental apparatus to protect the surrounding East Hall area from irradiation. It has been designed according to the maximum flux of $2.7 \times 10^{10}$ incident protons per second, in respect of the CERN safety regulations. Being the apparatus located in a fully enclosed area, it has become necessary to provide the experimental area with cooling and ventilation equipment to prevent overheating of detectors and electronics.

\section{Large Detectors upstream the magnet}

Two tracking devices have been installed in the secondary particle channel before the magnet and the collimator : the GEM/MSGC and the SFD. They are used to improve the resolution on the measurement of the longitudinal and transverse components of the relative momentum of pion pairs as determined by the drift chambers tracking system and the nominal position of the beam at the target center.

At the same time these detectors allow to select particle pairs originated by primary interactions at the target from the background of secondary interactions and particle decays. The ensemble of MSGC/GEM+SFD constitutes a tracking system with 7 detector planes with 2 stereo angles which provides adequate space resolution to reach the limit of multiple scattering in the target material.

To increase the detection capability on close-lying tracks, an Ionisation Hodoscope (IH) is installed downstream the SFD, with the purpose of detecting pion pairs with a too small opening angle to be resolved by the tracking detectors. This is achieved by a detailed pulse-hight analysis of the double ionisation produced by the particle pairs in 4 layers of scintillation counters.

A general picture of the above-mentioned detectors, as they are installed between the first vacuum chamber and the secondary particle channel, can be seen in Figure 5. 
Fig. 5. Photography of the three detectors installed upstream the magnet, between the first vacuum chamber (right-hand side) and the secondary particle channel. From right to left, the GEM/MSGC, SFD and IH detectors can be found. The primary proton beam line can be appreciated at the bottom.

\section{The GEM/MSGC detector}

This detector performs particle tracking at a distance of $2.4 \mathrm{~m}$ from the interaction point. It is a proportional gas detector, based on the principle of the Gas Electron Amplifier (GEM) [11] [12], complemented with a second amplification and readout stage provided by Micro Strip Gas Chambers (MSGC) [13] [14]. A more complete description of the detector and its performance is being prepared in a separate publication [15].

It measures particle coordinates in 4 planes along the direction of the incoming particle: $\mathrm{X}, \mathrm{Y}, \mathrm{U}, \mathrm{V}$, with orientations $0,90,5,85$ degrees, respectively, where the 0 degrees are defined by microstrips running vertically (X-coordinate). The stereo angles allow resolution of ghost combinations for two or more particles. With a single-hit space resolution close to $54 \mu \mathrm{m}$, this detector provides a precise measurement of the pion pair angular opening, ultimately limited by multiple scattering in the thin target.

\subsection{Detector concept}

Each chamber has active area $10.24 \times 10.24 \mathrm{~cm}^{2}$, and consists of a drift electrode, a GEM foil and a MSGC sensor. The GEM plane is evenly spaced from the other two with a uniform gap of $3 \mathrm{~mm}$, as indicated in Fig. 6. The drift electrode is made of a Chromium-coated thin glass $(200 \mu \mathrm{m})$. The GEM is a $50 \mu \mathrm{m}$ thick kapton foil copper-cladded on both sides with a $4 \mu \mathrm{m}$ thick $\mathrm{Cu}$ layer. The etching pattern is characterised by $50 \mu \mathrm{m}$ wide holes, $140 \mu \mathrm{m}$ apart [16]. Application of a potential difference of $400 \mathrm{~V}$ between the two metal layers $\left(V_{1}=-1600 \mathrm{~V}, V_{2}=-2000 \mathrm{~V}\right)$ produces electron amplification by a factor of 30 [12].

The MSGC sensor consists of $200 \mu$ m pitch alternating Chromium strips, with $9 \mu \mathrm{m}$ and $100 \mu \mathrm{m}$ anode and cathode width, respectively. They are implanted on a bare DESAG D263 substrate. Applied voltages are: -410 V on cathodes, and $-3000 \mathrm{~V}$ on the drift electrode, whereas anode strips are set to ground. The gas employed is a mixture of Ar-DME (60/40). Under these conservative conditions an overall detector gain of approximately 3000 is achieved. 


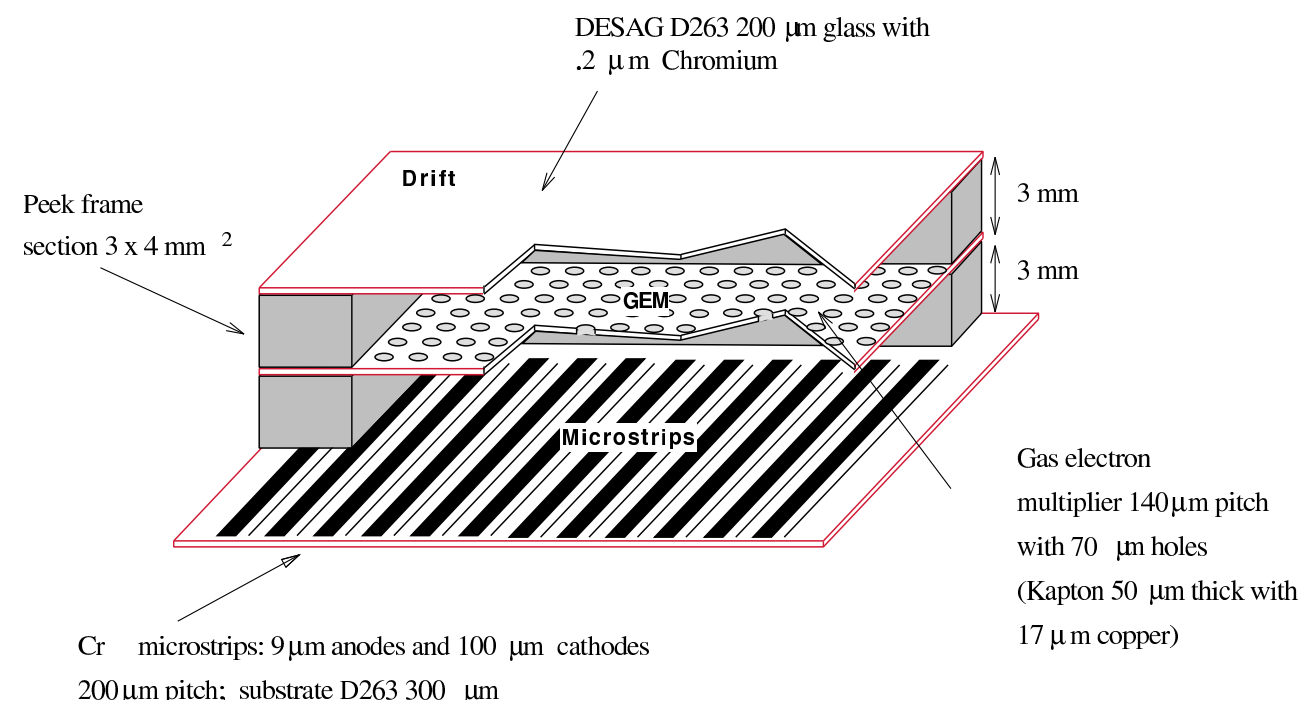

Fig. 6. Layout of the GEM/MSGC detector.

\subsection{Readout electronics}

It is composed of three parts: the analog boards, the control boards and the VME acquisition modules [17].

The analog boards contain the front-end electronics, and also serve as a mechanical support for the detector. They host 16 Analog Pipeline Chips (APC) [18], bonded on a multi-layer hybrid to the detector fan-out strips. Each APC has 64 inputs, with a charge sensitive preamplifier followed by analog pipeline of 32 capacitors, which is run at $10 \mathrm{MHz}$. In order to accelerate the serial readout, only 32 inputs are connected to the same chip. The analog pulse is formed by charge subtraction from consecutive capacitors, at positions given by the delayed trigger signal. This robust procedure, which allows easy integration of a large number of channels, limits however the time resolution of the readout to a window of approximately $200 \mathrm{~ns}$.

The control boards store the digital sequence needed for APC control and perform fast digital conversion (AD9048) of the 16 series of analog signals. These two functions are controlled by FPGA (Fast Programmable Gate Array) incorporated onto the board.

The VME modules handle the trigger signal and perform real time pedestal subtraction and zero suppression. One module is divided into 4 segments, each controlled by an independent FPGA. A Fast Clear function is implemented to be used in connection with the highest level of trigger (see trigger chapter), that stops the readout of current event and enables new triggers. 

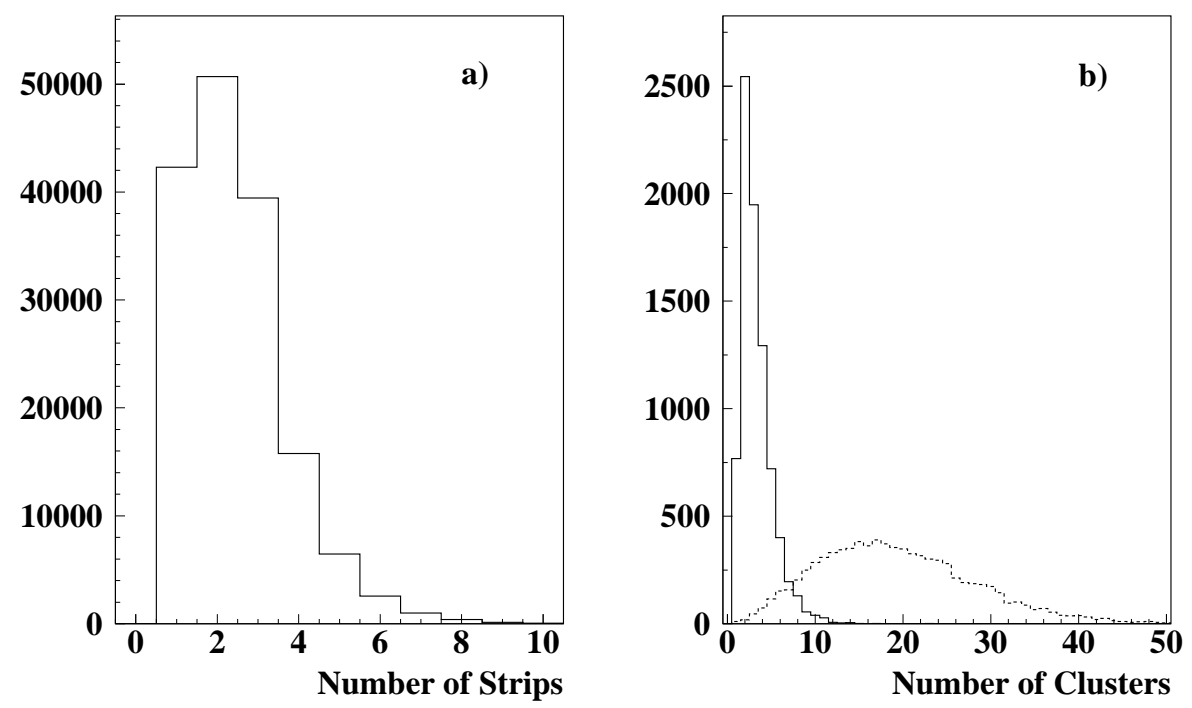

Fig. 7. a) GEM/MSGC strip multiplicity per hit-cluster b) cluster multiplicity per event in one plane $(\mathrm{X})$. The dotted line shows all clusters registered by the data acquisition, whereas the continuous line shows only those having a time tag, established when the cluster has a corresponding hit in the SFD aligned with the interaction point.

\subsection{Performance}

Neighbouring hit strips are pattern-recognised as clusters. The hit multiplicity in them (a) and the cluster multiplicity per plane (b) are shown in Fig. 7. Single hit resolution was determined during the commissioning run in April 2000 by setting all planes parallel to each other, and $54 \mu \mathrm{m}$ is a typical value, as shown in Fig. 8. Two detector planes were installed in 1999 and the full set in 2000. The number of detector dead channels is around $1 \%$ and no significant deterioration due to radiation has been observed since then. The average efficiency for a standard detector is $93 \%$. When at least 4 signals among 7 detectors are required to make a track, this is sufficient to provide $99 \%$ overall tracking efficiency upstream the magnet.

\section{The Scintillating Fibre Detector}

The Scintillating Fibre Detector (SFD), together with the GEM/MSGC detector, enables particle tracking to be performed upstream the magnet, with the required space and time resolution. In addition, it provides topological trigger capabilities [19] for rejection of pairs with relative distances larger than $9 \mathrm{~mm}$ at the detector location (this feature was used at the early stage of the experiment, see a footnote in section "Trigger system"). 

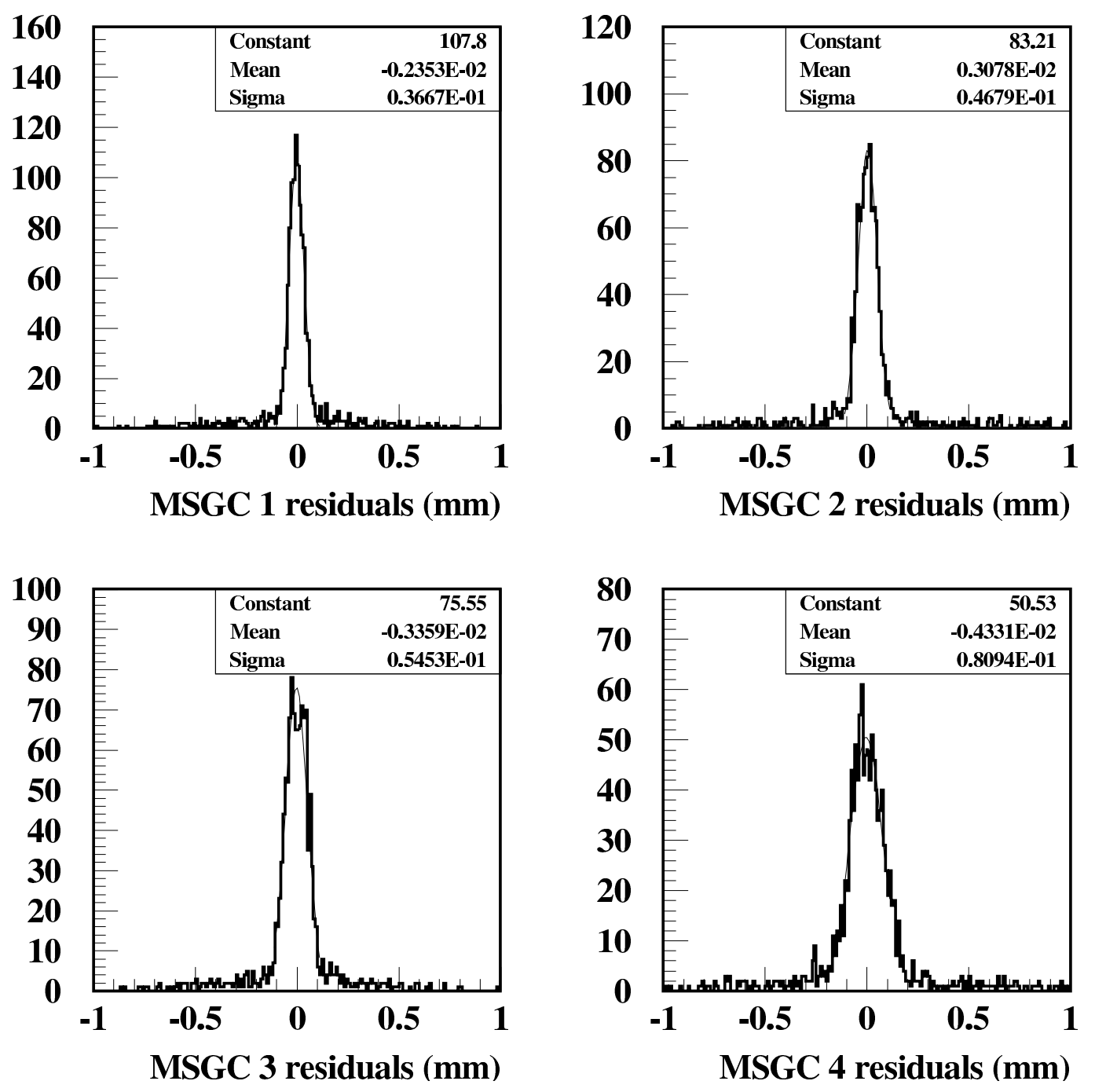

Fig. 8. Space resolution of the GEM/MSGC detector measured in a dedicated beam-test with 4 planes parallel to each other.

\subsection{Detector concept}

The SFD consists of three fibre planes to measure the $\mathrm{X}-$, Y- and U-coordinates of incident particles ${ }^{1}$. The SFD covers a $105 \times 105 \mathrm{~mm}^{2}$ area, each scintillating fibre (SciFi) array consists of several layers of KURARAY fibres. Five (for planes $\mathrm{X}$ and $\mathrm{Y}$ ) or three (for plane $\mathrm{U}$ ) fibres forming one sensitive column are mapped onto one channel of position-sensitive photomultiplier (PSPM). A layout illustrating the main characteristics of the SFD (for a plane with 5 fibres per column) and PSPM mapping is shown in Fig. 9.

1 The SFD operational in DIRAC until the end of 2001 run consisted of X and Y planes only. The U-plane described here was installed in early 2002. 


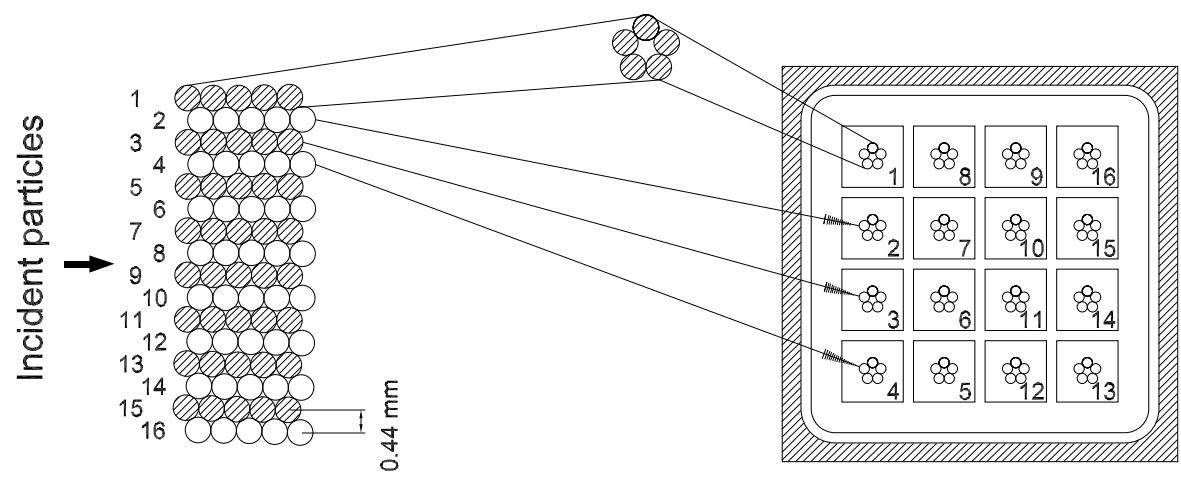

Fiber array

Fiber position on PSPM

Fig. 9. The SFD principal structure. A 16-channel fragment is shown.

The fibre columns pitch is $0.44 \mathrm{~mm}$, thus allowing some overlap to optimise efficiency. Each SciFi is connected by optical epoxy to a clear fibre light guide of $\sim 300 \mathrm{~mm}$ length. The edges of clear fibres, assembled in a bundle, are carefully polished and glued into the holes of a square black plate which fixes the fibre positions on the PSPM photocathode; no optical grease is used. The far end of the SciFi array is tightly connected to a mirror made of aluminised mylar. With this arrangement the light attenuation along the SciFi was found to be negligible. The details of the fibre arrays are listed in Table 2.

Table 2

SFD planes specification.

\begin{tabular}{|c|c|c|c|c|c|c|c|}
\hline & $\begin{array}{c}\text { Fibre } \\
\text { type }\end{array}$ & $\begin{array}{c}\text { Fibre } \\
\Phi(\mathrm{mm})\end{array}$ & $\begin{array}{c}\text { Length } \\
\mathrm{mm}\end{array}$ & $\begin{array}{c}\text { Fibres/ } \\
\text { column }\end{array}$ & $\begin{array}{c}X_{0} \\
\%\end{array}$ & $\begin{array}{c}\text { Num. of } \\
\text { channels }\end{array}$ & $\begin{array}{c}\text { Num. of } \\
\text { PSPM }\end{array}$ \\
\hline X & SCSF38 & 0.50 & 130 & 5 & 0.8 & 240 & 15 \\
Y & SCSF38 & 0.50 & 130 & 5 & 0.8 & 240 & 15 \\
U & SCSF78M & 0.57 & 150, & 3 & 1.0 & 320 & 20 \\
& & & 130,70 & & & & \\
\hline
\end{tabular}

The SFD individual planes are installed close to each other. Direction of fibres is orthogonal in planes $\mathrm{X}$ and $\mathrm{Y}$ while plane $\mathrm{U}$ is rotated by 45 degrees. The U-plane has 5 sections of different fibre lengths to cover roughly the same area like the $\mathrm{X}$ - and Y-planes.

A 16-channel metal dynode position-sensitive photomultiplier tube, Hamamatsu H6568, has been selected as photosensor. This photomultiplier is characterised by good timing properties (rise time $\sim 0.7 \mathrm{~ns}$ ), low noise (1-2 pulses/s at a nominal detection threshold) and perfect single photoelectron spectrum. It has been modified to monitor the amplitude from the last dynode for calibration purposes. The level of optical cross talk among the PSPM channels was found to be $\sim 1 \%$ (with a $1.6 \mathrm{~mm}$ diameter light spot on the photocath- 
ode). The measured linear range of this tube (for the linear bleeder) extends up to 15 photoelectrons at $950 \mathrm{~V}$.

The detector concept with almost independent channels was developed in the context of the RD-17 project [20]. The results from the SFD study with a test beam of low intensity, reported in [21], are the following:

- light output 6-10 photoelectrons,

- average detection efficiency $98.4 \%$,

- r.m.s. of the detection efficiency $1 \%$,

- average hit multiplicity 1.1 ,

- spatial resolution $(\sigma) 127 \mu \mathrm{m}$,

— time resolution $(\sigma) 0.65 \mathrm{~ns}$.
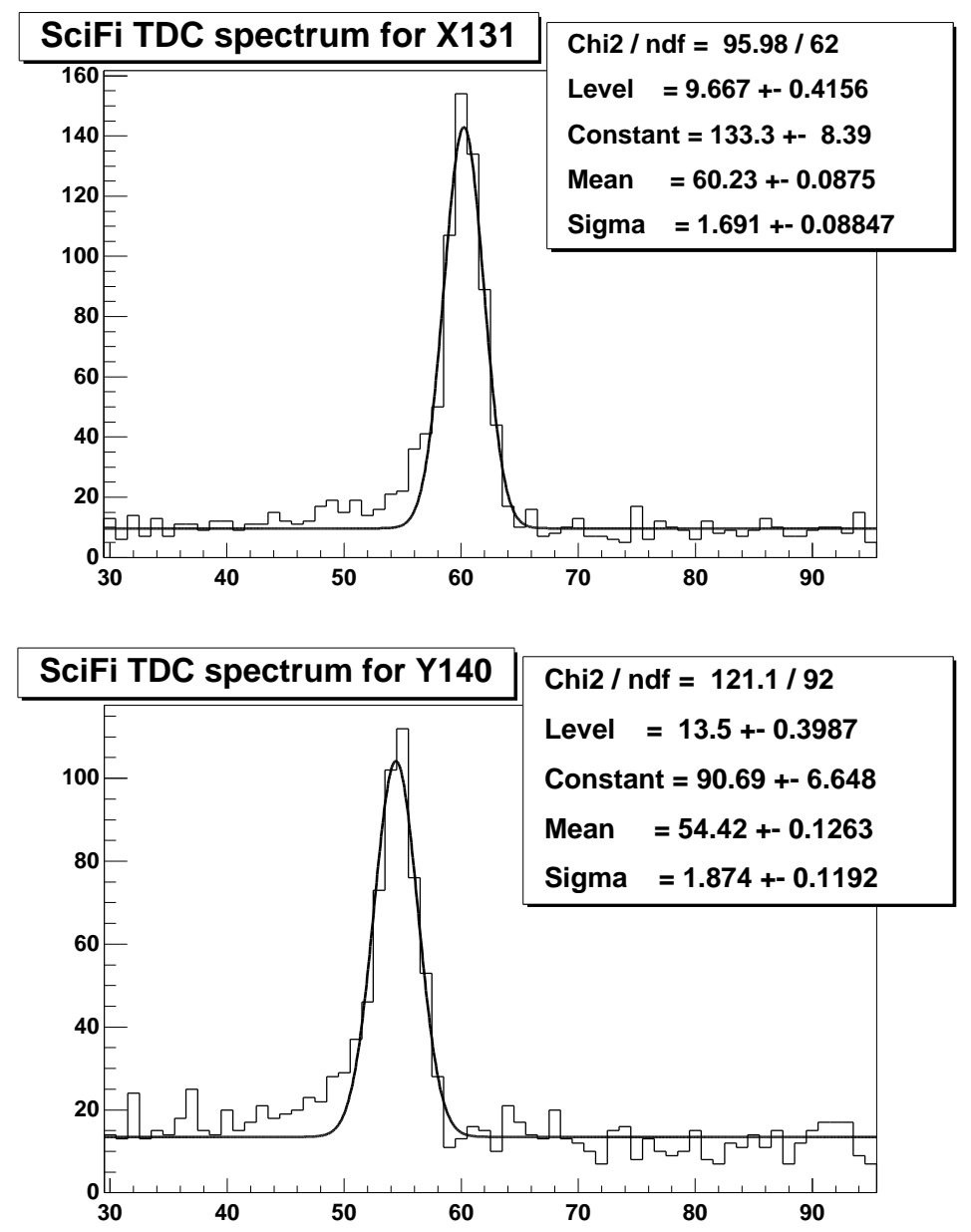

Fig. 10. SFD raw time spectra for $e^{+} e^{-}$trigger data. Here and in Fig. 11 the horizontal scale is in TDC channels, the channel width is $0.5 \mathrm{~ns}$. 


\subsection{Readout electronics}

A dedicated electronic circuit (PSC) has been custom developed to provide signal discrimination with dynamic rejection of cross-talk in adjacent channels using the peak-sensing technique. A detailed description of a 32-channel PSC module is given in [22]. Discrimination of a channel is given by the condition $2 A_{i}-A_{i-1}-A_{i+1}>A_{t h r}$, where $A_{i}$ are channel signal amplitudes and $A_{t h r}$ defines the threshold value. The PSC algorithm provides efficient detection of double tracks from time correlated particle pairs (up to $\sim 5$ ns time difference) when the relative distance between the two tracks is larger than the fibre column pitch. However, when adjacent fibre columns are crossed by two particles simultaneously, then the PSC algorithm leads to a suppression (by $20-40 \%$ ) of the detected yield of double track events. In such cases a signal is detected for only one of two hit columns. The detection efficiency depends on the detector light output in a specific channel. For particle pairs with relative time differences greater than $10 \mathrm{~ns}$ (accidental pairs) the PSC behaves as an ordinary leading edge discriminator.
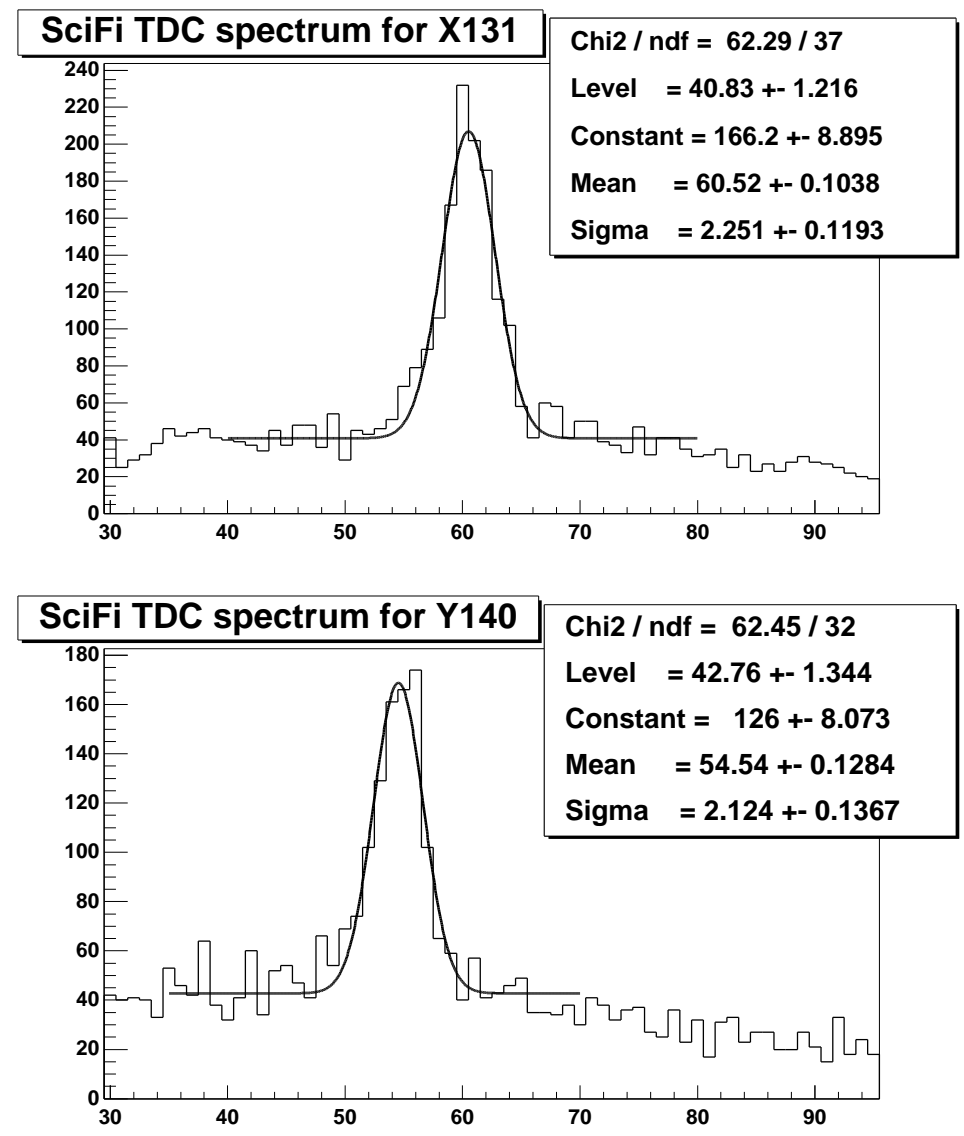

Fig. 11. SFD raw time spectra for $\pi^{+} \pi^{-}$trigger data.

The front-end electronics is located inside the beam area close to the detector. The PSC output ECL signals are sent to LeCroy 3377 multi-hit TDC. This 
solution does not require additional delay lines to adjust the timing of the SFD with respect to the trigger timing.

\subsection{Performance in the experiment}

Due to the high flux of particles at the position of the detector close to the target, and to the presence of a non-negligible amount of inclined tracks associated with secondary interactions in the channel, the SFD performances in the experiment slightly differ from the above mentioned. The detection efficiency is still high (around 98\%), but the average hit multiplicity is near 5 in the $50 \mathrm{~ns}$ time window of TDC (at a nominal beam intensity of $10^{11}$ protons per spill impinging on a $94 \mu \mathrm{m}$ Ni target). The raw time spectra, obtained from $e^{+} e^{-}$and $\pi^{+} \pi^{-}$events, are shown in Fig. 10 and Fig. 11, respectively, for two arbitrary SFD channels. The width of the distributions is dominated by the time jitter of the trigger signal. After off-line deconvolution of the trigger time jitter the resolution of the SFD is found to be $\sigma=0.8 \mathrm{~ns}$.

\section{The Ionisation Hodoscope}

Charged pions originated from pionium breakup cross the upstream detectors at rather small relative distances. When the distance is less than the double track resolution of the upstream tracking devices, then only one hit is detected, thus making the event reconstruction ambiguous. That is why another technique based on a measurement of the ionisation loss is used as well.

A dedicated Ionisation Hodoscope (IH) [23] has been built to separate double ionisation signals produced by close pion pairs incident on the same scintillating slab, from single ionisation signals produced by one particle. In this way, the uncertainties resulting from the inefficiency in detecting two tracks with relative distance approaching zero can be significantly reduced.

The Ionisation Hodoscope described here was installed in 2001 to replace a previous version of a similar detector type [24], consisting of only two planes with 16 slabs of $2 \mathrm{~mm}$ thickness, oriented in the vertical direction.

The present IH detector is a scintillation hodoscope consisting of 4 planes of $11 \times 11 \mathrm{~cm}^{2}$ sensitive area placed normally to the axis of the setup (Fig. 12), $3 \mathrm{~m}$ downstream the target. Two planes have vertically oriented slabs (planes X-A and $\mathrm{X}-\mathrm{B}$ ) whereas the other two have horizontal slabs (planes $\mathrm{Y}-\mathrm{A}$ and $\mathrm{Y}-\mathrm{B}$ ). They are arranged in the following sequence, moving along the beam direction: 


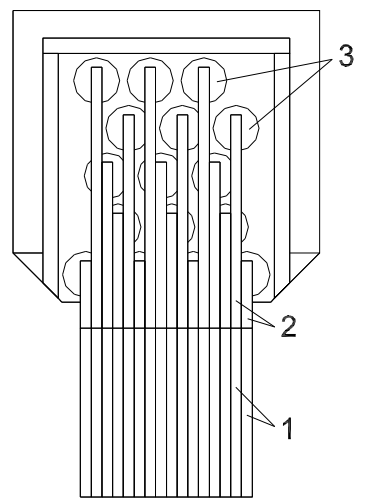

Fig. 12. Design of the IH scintillation plane. 1 - scintillators, 2 - light-guides, 3 - PM photocathodes.

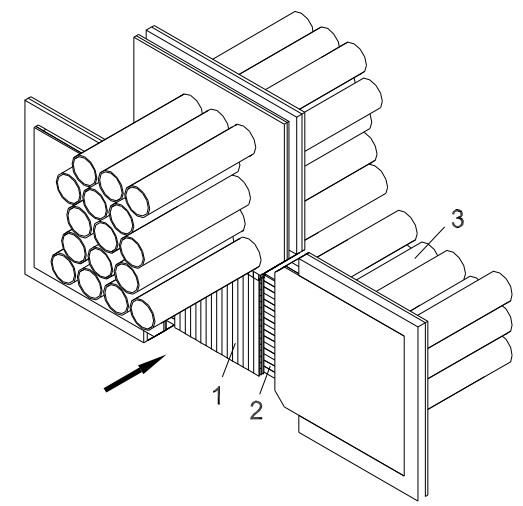

Fig. 13. Isometric view of the Ionisation Hodoscope. 1 - scintillators, 2 - light-guides, 3 - photomultipliers with shielding.

$\mathrm{X}-\mathrm{A}, \mathrm{Y}-\mathrm{A}, \mathrm{X}-\mathrm{B}, \mathrm{Y}-\mathrm{B}$. This ordering has been chosen to minimise possible crosscorrelations between signals in the planes (e.g. due to $\delta$-electrons). Each plane is assembled from 16 plastic scintillating slabs made of fast scintillator (BC408). Planes with the same slab orientation are shifted by a half-slab-width with respect to each other. The slabs are $11 \mathrm{~cm}$ long, $7 \mathrm{~mm}$ wide and $1 \mathrm{~mm}$ thick. They are connected to the PM photocathodes via $2 \mathrm{~mm}$ thick and $7 \mathrm{~mm}$ wide lucite light guides (fig. 13).

The front and rear surfaces of a slab are covered by a millipore film [25] for efficient light collection. At the lateral surface of the slab, light is reflected by a thin $(30 \mu \mathrm{m})$ aluminised black mylar film, which is used instead of millipore film in order to minimise the gaps between adjacent slabs. A typical gap between two adjacent slabs in this configuration is less than $70 \mu \mathrm{m}$ wide.

Scintillation light is detected by FEU-85 photomultipliers with $25 \mathrm{~mm}$ diameter photocathodes. Photomultipliers are assembled by 16 units into a compact set, allowing independent replacement of each PM. Photocathodes are in optical contact with the wide side of a light guide instead of the traditional butt-end readout. This improves the light collection efficiency by about $50 \%$. 


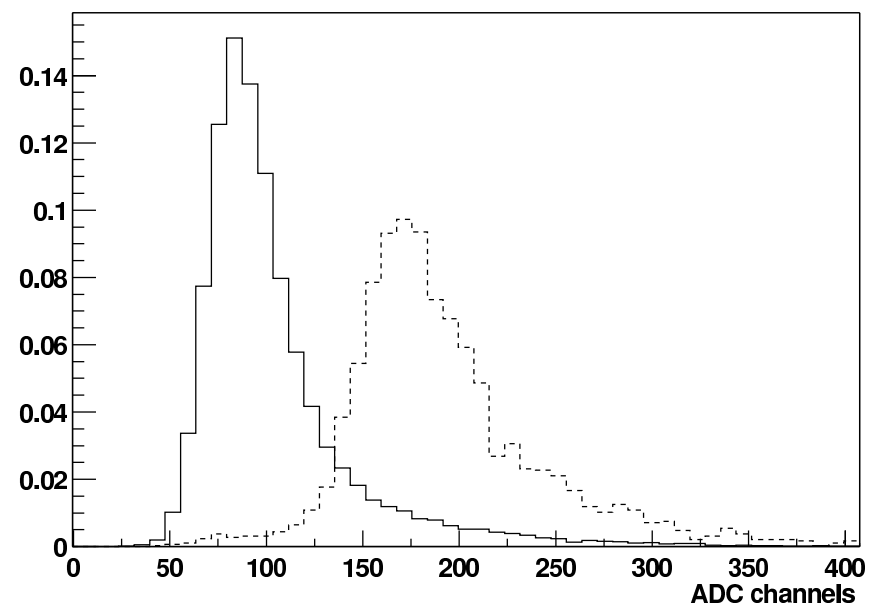

Fig. 14. Typical ADC spectra for single (solid line) and double (dashed line) ionisation loss from particles crossing one IH scintillating slab.

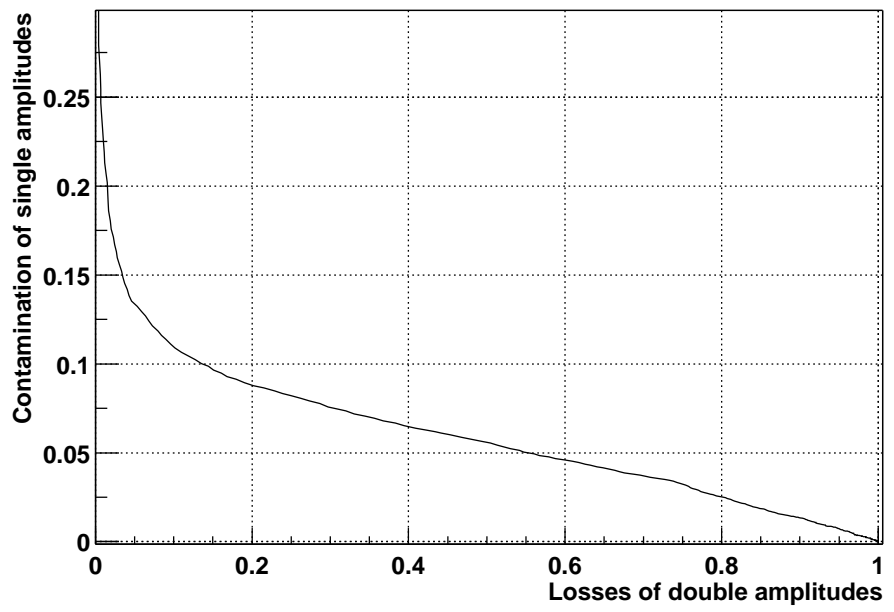

Fig. 15. Contamination of single ionisation amplitudes as a function of losses of double ionisation as obtained from the analysis of the spectra of Fig. 14.

As the detector is highly loaded by intense particle flux, the last 4 PM-dynodes are fed by an additional power supply to ensure a constant PM amplification throughout the spill.

Signal amplitude and time are digitised by LeCroy ADC 4300B and TDC 3377 modules, respectively. The time resolution of the IH detector is better than 1 ns. The typical response of one IH channel to close particle pairs incident on one scintillating slab and to single particle is shown in Fig. 14. If a threshold is set to retain $90 \%$ of the double ionisation signal from pairs, the contamination from single particle amplitudes is less than $15 \%$ (Fig. 15). 


\section{Drift Chambers}

\subsection{General layout and characteristics}

The drift chamber system is used to perform particle tracking downstream the dipole magnet. The system is designed to sustain a high particle fluency in the secondary channel, reaching $10 \mathrm{kHz} / \mathrm{cm}^{2}$ at the innermost region.

A two-arm solution has been chosen, except for the first chamber which is a single large module (DC-1) designed with two separated sensitive areas $0.8 \times$ $0.4 \mathrm{~m}^{2}$ each. This chamber provides 6 successive measurements of the particle trajectory along the coordinates $\mathrm{X}, \mathrm{Y}, \mathrm{W}, \mathrm{X}, \mathrm{Y}, \mathrm{W}$, where $\mathrm{W}$ is a stereo angle with inclination $11.3^{\circ}$ with respect to the $\mathrm{X}$-coordinate. DC-1 is instrumented with 800 electronic channels.

Each of the two arms consists of 3 chamber modules, of identical design, measuring coordinates X,Y (DC-2), X,Y (DC-3) and X,Y,X,Y (DC-4) following the direction of the outgoing particle. Their dimensions are $0.8 \times 0.4 \mathrm{~m}^{2}$ (DC2), $1.12 \times 0.4 \mathrm{~m}^{2}$ (DC-3), and $1.28 \times 0.4 \mathrm{~m}^{2}$ (DC-4). Both arms together contain 1216 electronic channels.

The distance between the center of the first half of DC1 and the center of DC4 provides a lever-arm of $1.6 \mathrm{~m}$ along the average particle path, having uniform spacing of chambers DC-2 and DC-3 along this path. Characteristics of the drift chamber system are summarised in Table 3.

\subsection{Drift chamber electrodes}

A schematic drawing of the sensitive element is shown in Fig. 16. The anode wires pitch is $10 \mathrm{~mm}$, the distance $\mathrm{L}$ between the anode and cathode planes is $5 \mathrm{~mm}$. The cathode planes and potential wires are at equal voltages. As seen in the figure, a sensitive area, corresponding to each anode wire and limited by the cathode planes and potential wires, has a square $\left(10 \times 10 \mathrm{~mm}^{2}\right)$ shape. In this case, with a suitable gas mixture, it is possible to achieve a linear behaviour of the drift function, except in a small region near the potential wire.

Cathode planes are made of $20 \mu \mathrm{m}$ thick carbon-coated mylar foils with a surface resistivity of about $400 \Omega$ per square. Such cathode foils provide stable chamber operation due to a high work function of the carbon coating and, being thin, add only small amount of material along the particle path. 
Table 3

General properties of the DC modules.

\begin{tabular}{|c|c|c|c|}
\hline $\begin{array}{c}\text { Module } \\
\text { type }\end{array}$ & $\begin{array}{l}\text { Sensitive } \\
\text { area, } \mathrm{cm}^{2}\end{array}$ & $\begin{array}{l}\text { Measured } \\
\text { coordinate }\end{array}$ & $\begin{array}{c}\text { Number of } \\
\text { planes }\end{array}$ \\
\hline \multirow[t]{6}{*}{ DC-1 } & \multirow{3}{*}{$\begin{array}{l}40 \times 80 \\
\text { left arm }\end{array}$} & $X$ & 2 \\
\hline & & $Y$ & 2 \\
\hline & & $W$ & 2 \\
\hline & \multirow{3}{*}{$\begin{array}{l}40 \times 80 \\
\text { right arm }\end{array}$} & $X$ & 2 \\
\hline & & $Y$ & 2 \\
\hline & & $W$ & 2 \\
\hline \multirow[t]{2}{*}{ DC-2 } & \multirow[t]{2}{*}{$40 \times 80$} & $X$ & 1 \\
\hline & & $Y$ & 1 \\
\hline \multirow[t]{2}{*}{ DC-3 } & \multirow[t]{2}{*}{$40 \times 112$} & $X$ & 1 \\
\hline & & $Y$ & 1 \\
\hline \multirow[t]{2}{*}{ DC-4 } & \multirow[t]{2}{*}{$40 \times 128$} & $X$ & 2 \\
\hline & & $Y$ & 2 \\
\hline
\end{tabular}

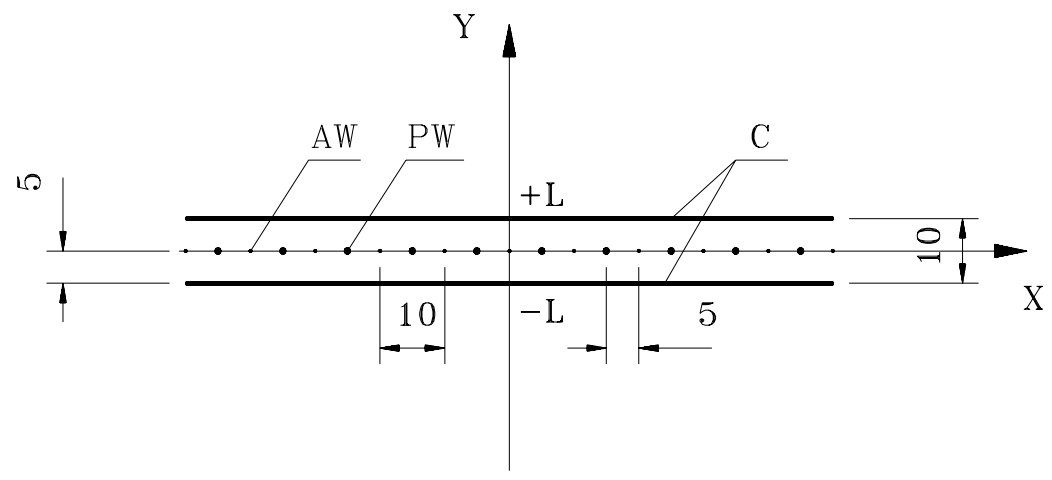

Fig. 16. Schematic view of the wire chamber electrodes: $A W$ - anode wires, $P W-$ potential wires, $C$ - cathode foils. Dimensions are in $\mathrm{mm}$.

Anode and potential wires of $50 \mu \mathrm{m}$ and $100 \mu \mathrm{m}$ diameter, respectively, are made of a copper-beryllium alloy. The rather large diameter of the anode wires has been chosen in order to operate the chambers at high current avalanche amplification mode. 


\subsection{Chamber design}

The chamber design is shown in Fig. 17 for the case of the DC-2 module. The module is a stack of aluminium and fibreglass frames, each of $5 \mathrm{~mm}$ thickness, fixed by screws. The fibreglass frames are the supports for the chamber electrodes (anode and potential wires and cathode foils). The two outer aluminium frames in the stack are used to fix the mylar window, and the inner ones are the spacers between the fibreglass frames. Rigidity of the module is enforced by aluminium rectangular tubes screwed to the surface of the frame package.

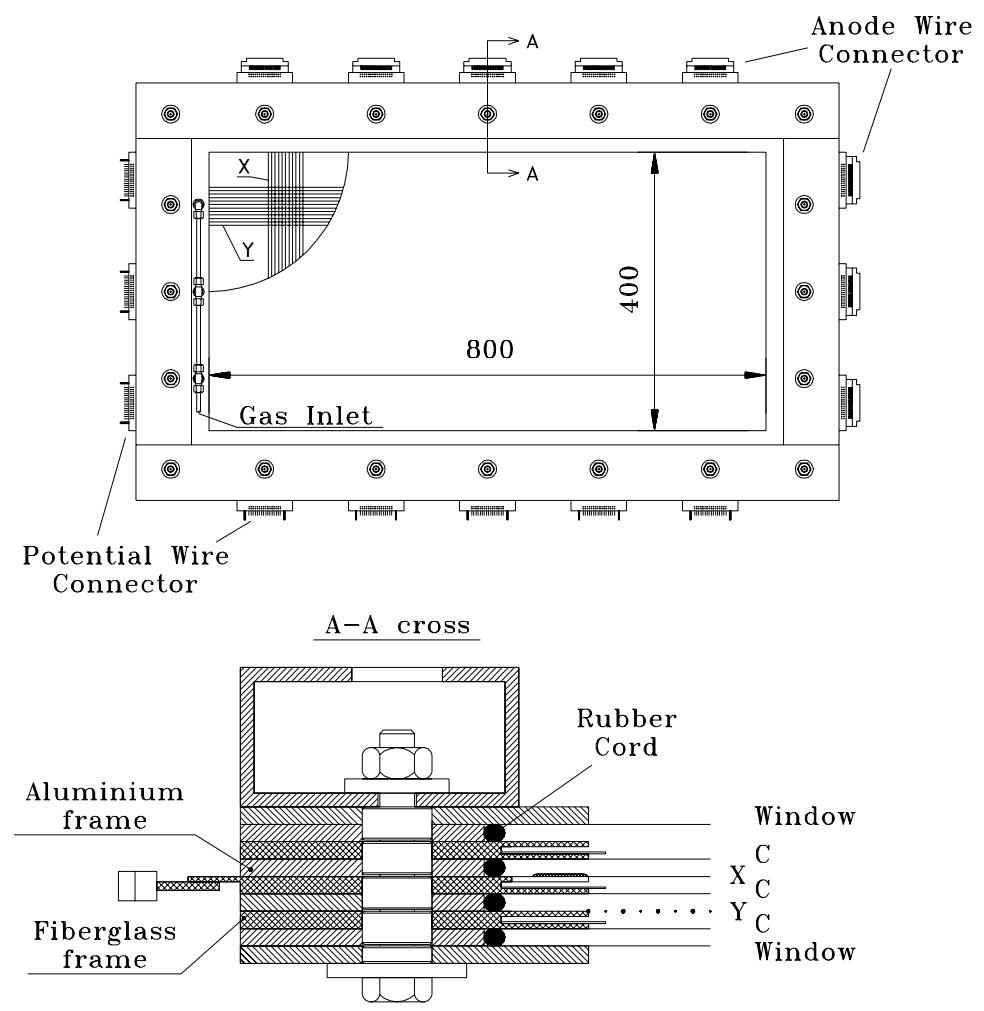

Fig. 17. Design of the DC-2 module. Upper figure: general view. Lower figure: structure of the frame stack; $\mathrm{X}-X$-plane, $\mathrm{Y}-Y$-plane, $\mathrm{C}$ - cathode foils.

Gas tightness of the chamber module is provided by rubber o-rings glued along the inner edges of the aluminium frames. Within a module, gas flows sequentially in the sub-volumes defined between cathode foils, by means of holes drilled on opposite sides of the fibreglass frames.

The design of module DC-1 differs from the one shown in Fig. 17. The main difference, illustrated in Fig. 18, consists in the fact that DC-1 comprises, in a single gas volume, two sets of sensitive planes, placed symmetrically to the left and right hand side of the spectrometer axis. The middle zone, which is strongly irradiated by particles (mostly fast protons from target fragmentation), is made insensitive to the particle flux. The limiting edge of the sensitive 
zones, close to the axis, can be varied. This is possible by means of a stripped structure of the neighbouring cathodes, which allows stepwise application of voltage. This design of the DC-1 module ensures little amount of material, by avoiding frames in the small angle region.

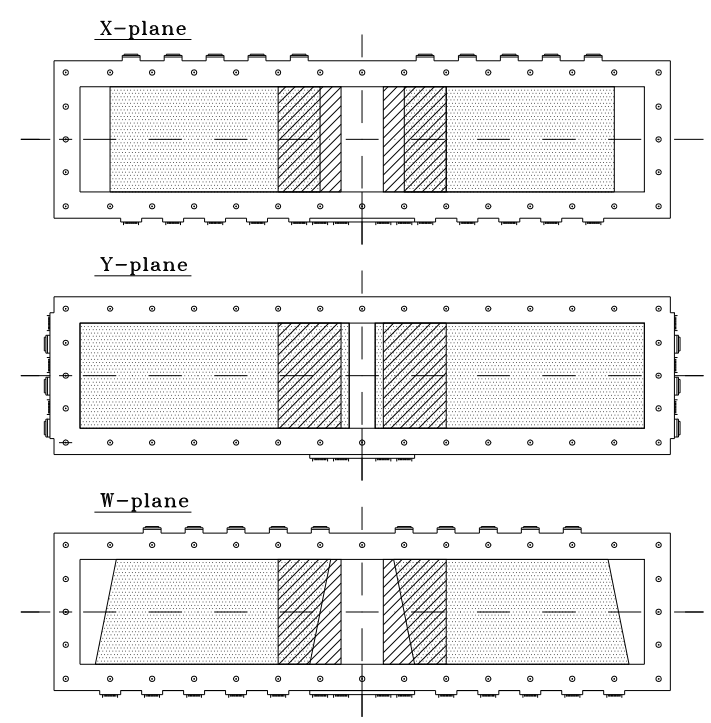

Fig. 18. Schematic view of the $D C-1$ module. Dotted areas show the sensitive regions of the $X$-, $Y$ - and $W$-planes. Hatched areas mark the zones of the cathode strips which allow to change the width of the insensitive area in the central region.

\subsection{Chamber operation and performance}

The drift chambers operate in a high current avalanche mode. This mode is characterised by high pulse amplitude (about $1 \mathrm{~mA}$ ), small pulse width (20 ns), and stable operation due to an efficiency plateau larger than $1 \mathrm{kV}$. The single hit efficiency is above $96 \%$ when the particle flux is about $10 \mathrm{kHz} / \mathrm{cm}^{2}$. The employed gas mixture is $\mathrm{Ar}(\sim 50 \%)+i \mathrm{C}_{4} \mathrm{H}_{10}(\sim 50 \%)+\mathrm{H}_{2} \mathrm{O}(0.5 \%)$, and the chamber operation voltage is $3.85 \mathrm{kV}$.

A space-to-time relationship was extracted from the time spectrum and its integral distribution shown in Fig. 19, for a sample of clean events with a small amount of background hits. The integral distribution has been parameterised by a second order polynomial of the type:

$$
l=a_{1} \times t^{\star}+a_{2} \times t^{\star 2} .
$$

In this formula $t^{\star}=t_{T D C}-t_{0}-\delta t$, where $\delta t$ is the signal propagation time along the anode wire.

Study of the drift function parameters for different chamber planes at different beam intensities shows good stability of the above relation. For this reason the 
same drift function parameters $\left(a_{1}\right.$ and $\left.a_{2}\right)$ were used for all chamber planes during the off-line track reconstruction procedure.
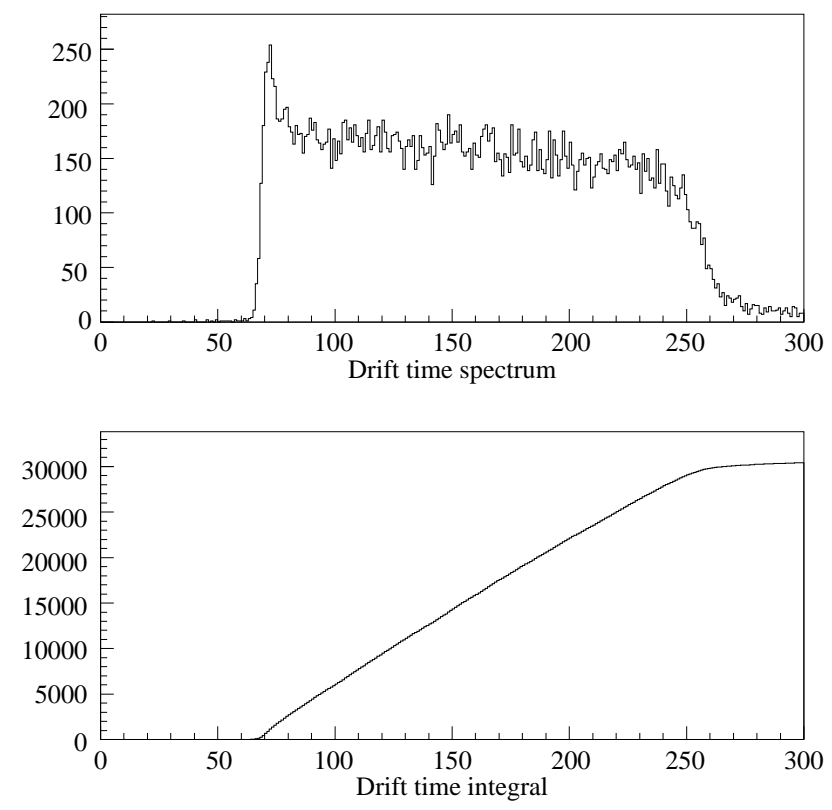

Fig. 19. Distribution of the drift time (upper) and its integral spectrum (lower) for the $X 4$-plane. Horizontal scale is in TDC channels, bin width is $0.5 \mathrm{~ns}$.

Coordinate resolution of the DC system is illustrated in Fig. 20, where the distribution of differences between the predicted position and measured coordinates in one of the planes is shown (X4-plane right arm). The measured standard deviation, $\sigma=100 \mu \mathrm{m}$, is defined not only by the intrinsic chamber plane resolution, but also by the accuracy of the predicted track coordinates. Taking the latter into account the measured intrinsic space resolution of one plane is better than $90 \mu \mathrm{m}$.

Tracking efficiency of the drift chamber system as a whole is about $99 \%$, due to the fact that the requested number of hits per reconstructed track is less than the total number of sensitive planes crossed by a particle.

\subsection{Readout electronics}

The readout electronics of the drift chambers, which is a custom-made system [26], provides data readout into the data collection memories and input to the trigger processor (see section "Trigger system").

The sensitive wire signals are digitised in the 16-channel multi-hit time-todigital converter boards (TDC), which are plugged in the connectors mounted onto the chamber frames. This solution results in reduced number of electronic 


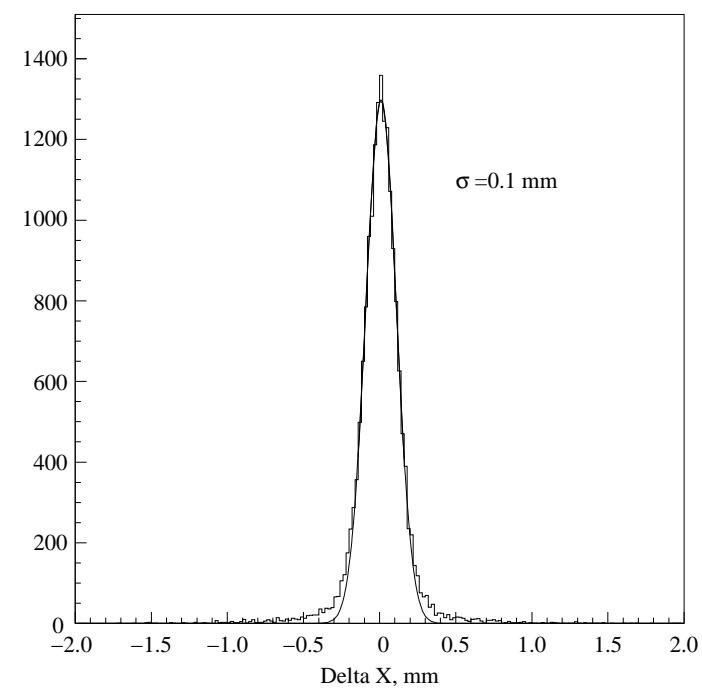

Fig. 20. Distribution of differences between the measured and predicted $\mathrm{X}$-coordinate for one drift chamber plane $(X 4)$.

units, small number of cables and high noise immunity. The detection threshold in the TDC board can vary from 0.05 to $2 \mathrm{~mA}$, the maximum number of hits per channel is 16 . Least count of TDC is $0.5 \mathrm{~ns}$, which corresponds to a drift distance of $25 \mu \mathrm{m}$ at a constant drift velocity of $50 \mu \mathrm{m} / \mathrm{ns}$, well below the chamber intrinsic space resolution.

A complete readout chain consists of TDC boards, bus drivers, readout controller and VME memory. Up to 8 TDC boards can be connected to the bus driver via common data and control buses, forming a segment of the DC readout system. Similarly, up to 8 segments are connected to a readout controller forming a readout branch. The data of an event are stored in local data buffers until the higher level trigger decision is issued. If the event is accepted, the data are serially transferred via the readout controller to the VME memories. To read out all the DC data 3 readout branches are used. The accepted data are transferred to the VME buffers within $5 \mu$ s on average. The DC readout is fast enough compared to the global readout time of the experiment.

For trigger purposes the DC readout system is equipped with fast data ports which directly transmit the hit wire numbers to a trigger track processor. This provides a minimum access time to the data, thus reducing the latency of the trigger system. Transmission to the track processor is performed in parallel. 


\section{The Vertical Hodoscopes (Time-of-Flight De- tector)}

The vertical hodoscope (VH) consists of an array of vertical scintillating slabs placed downstream the DC system. The VH system, together with the horizontal hodoscope, provides fast coincidence signals between the spectrometer arms necessary for the first level trigger. It is also used, in correlation with other detectors, in the definition of dedicated triggers for calibration purposes and of a higher level trigger for the selection of low $Q$ events (see trigger section later).

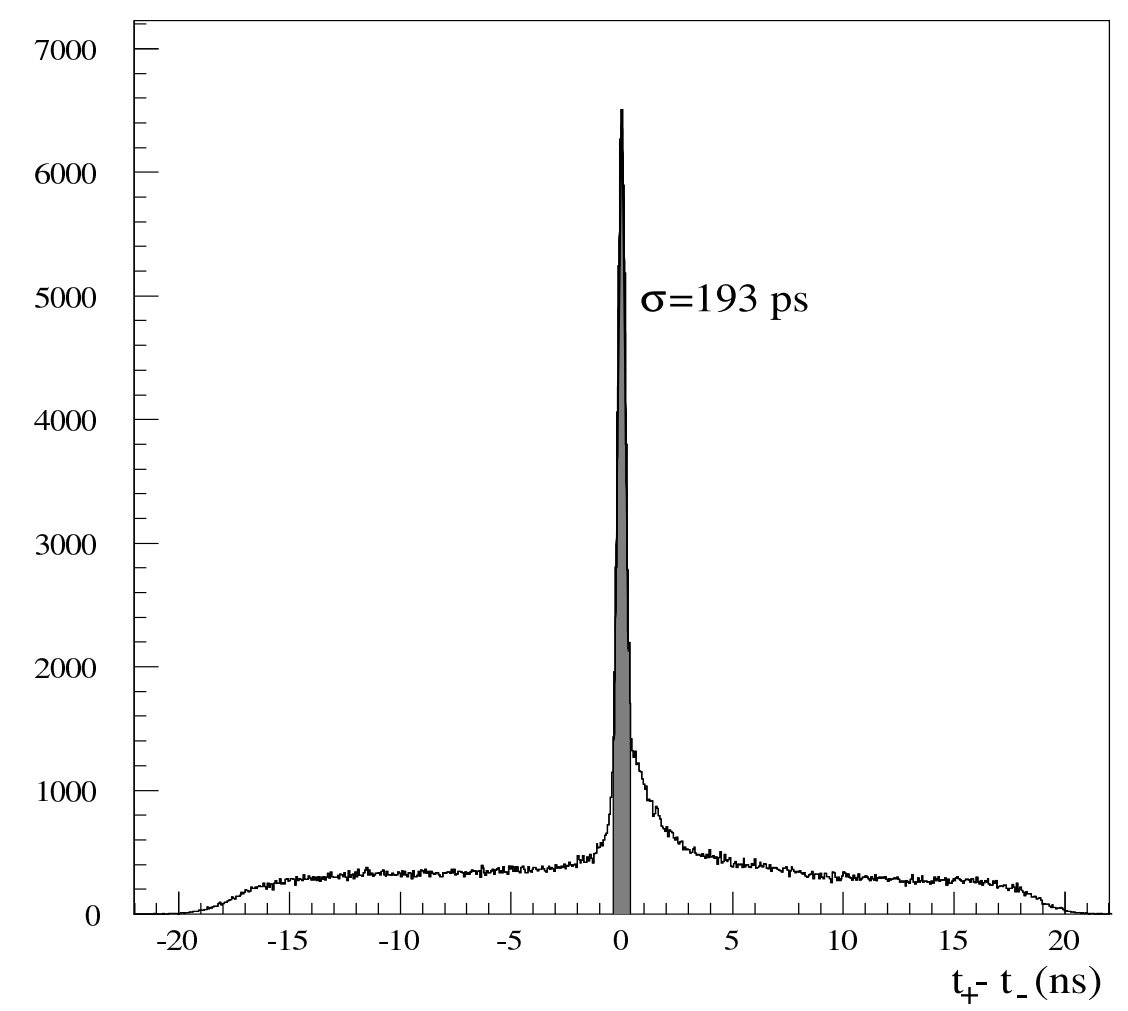

Fig. 21. Time difference between charged positive and negative particles obtained from standard hadron trigger data. The central peak has a gaussian width of $193 \mathrm{ps,}$ and the shaded area represents a $2 \sigma$ cut used to select prompt $\pi \pi$ events. The flat background is originated from accidental pairs, not belonging to the same beam interaction. Note the shoulder on the right-hand side of the peak, due to $\pi^{-} p$ prompt pairs.

A key function of this detector, which motivated a special design, is to provide a very accurate time definition of pion pairs originated from the same proton interaction (prompt pairs), in order to perform a clean separation (in off-line analysis) with respect to pairs in which the pions are produced at different times (accidental pairs). 
Used as a time-of-flight detector it allows to identify $p \pi^{-}$pairs in prompt events, as they might constitute a significant source of background to the $\pi^{+} \pi^{-}$signal.

The VH detector consists of two identical telescopes matching the acceptance of the drift chamber system previously described. Each telescope contains an array of 18 vertical scintillation counters. The scintillating material is BICRON BC420 and the slab dimensions are $40 \mathrm{~cm}$ length, $7 \mathrm{~cm}$ width and $2.2 \mathrm{~cm}$ thickness. Scintillation light is collected at both ends by two 12-dynode Hamamatsu R1828-01 photomultipliers (chosen because of their small transition time spread) coupled to fish-tail light guides. Voltage dividers were designed to provide sufficient bleeder current at particle rates up to $2 \times 10^{6}$ $\mathrm{Hz}$, while preventing any degradation of the time resolution.

The front-end electronics was designed to minimise the time jitter. This is achieved by using LeCroy L3420 constant fraction discriminators, followed by CAEN C561 meantimers to provide a position independent time measurement. Hodoscope signals are delayed and transferred to the data acquisition system using shielded twisted-pair cables. Time digitisation is performed by LeCroy 4303 time-to-FERA Converter followed by ADC 4300B. The least count of this ensemble is 62 ps.

The VH single-hit detection efficiency is $99.5 \%$ for the positive, and $98.8 \%$ for the negative hodoscope arms. In Fig. 21 the distribution of the time difference between positive and negative pions in the spectrometer is shown. The observed ratio between prompt and accidental pairs in the $2 \sigma$ cut region around the peak is about 16 .

The overall time resolution of the system has been measured with $e^{+} e^{-}$pairs to be 127 ps per counter [27], which corresponds to 174 ps accuracy for the time difference between positive and negative arms (time-of-flight resolution). The latter is shown in Fig. 22.

The dedicated $e^{+} e^{-}$calibration trigger selects $e^{+} e^{-}$pairs from $\gamma$ conversions and Dalitz decays of $\pi^{0}$ which are almost synchronous in time, as the time of flight of $e^{+} e^{-}$pairs is momentum independent in the available setup range of momenta.

This timing capability allows to separate $\pi^{+} \pi^{-}$from $\pi^{-} p$ pairs in the momentum range from 1 to $5 \mathrm{GeV} / c$, and from $\pi K$ pairs in the range from 1 to $2.5 \mathrm{GeV} / c$, as illustrated in Fig. 23. 


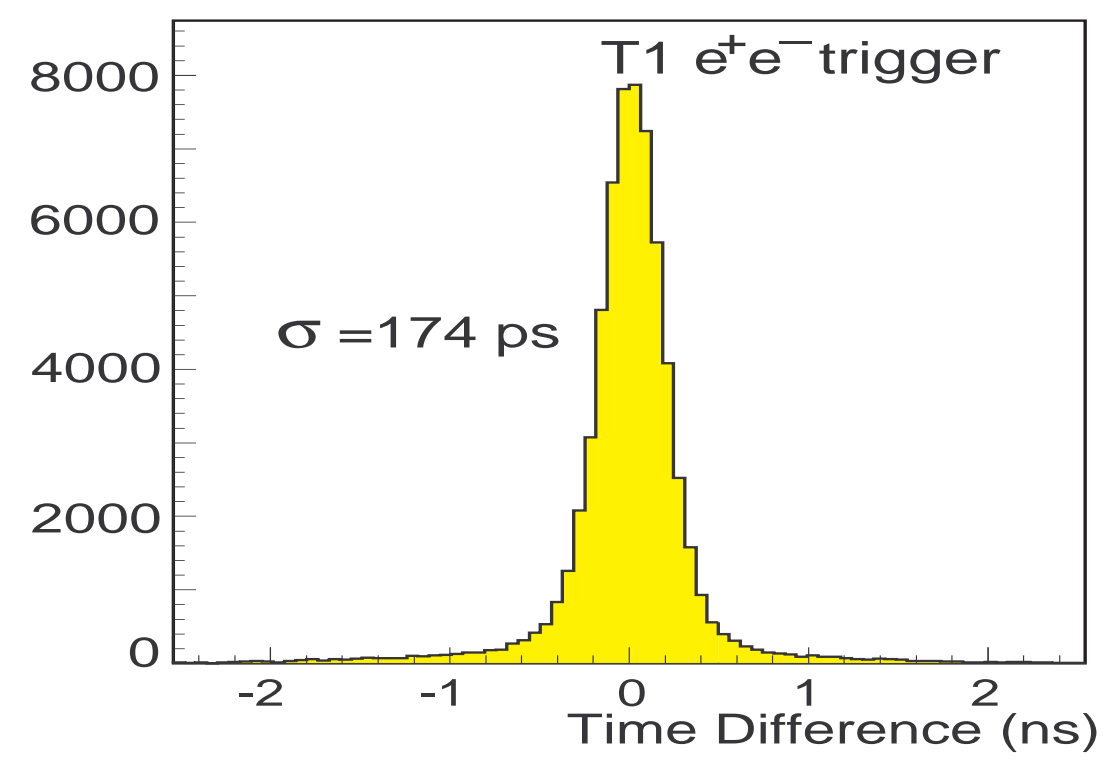

Fig. 22. Time difference spectrum for $e^{+} e^{-}$pairs detected by the vertical hodoscopes, after path length correction. Data come from a sample of $e^{+} e^{-}$calibration triggers.

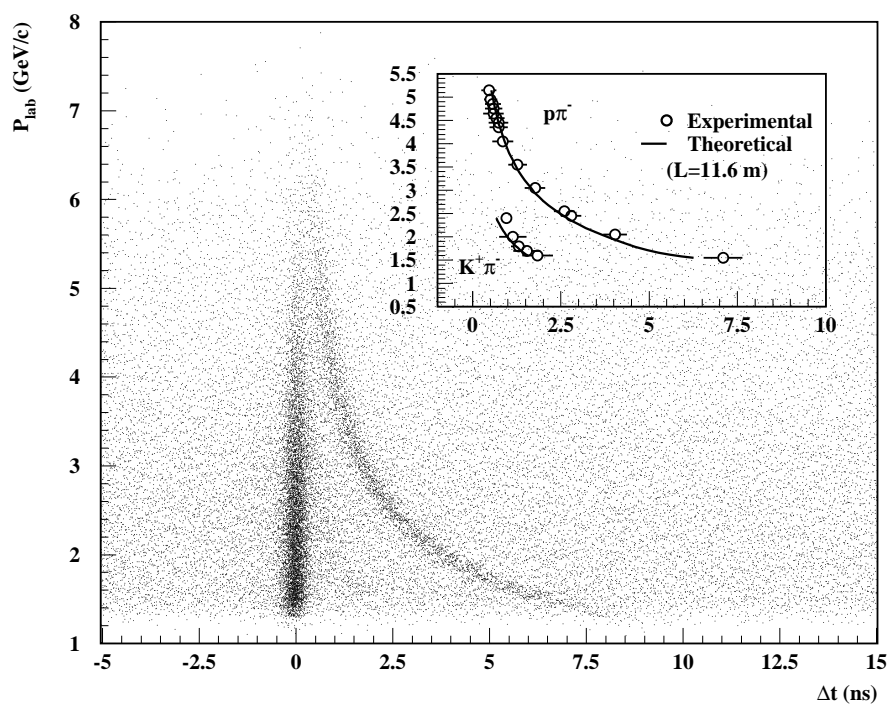

Fig. 23. Correlation between the measured momentum of the positive particle and the VH time difference between the positive and negative spectrometer arm, taking into account the correction for the difference in path length. The accumulation bands correspond to $\pi^{-} \pi^{+}$(vertical band) and $\pi^{-} p$ (curved band) pairs. A small cluster of $\pi^{-} K^{+}$pairs is also visible in the intermediate region. 


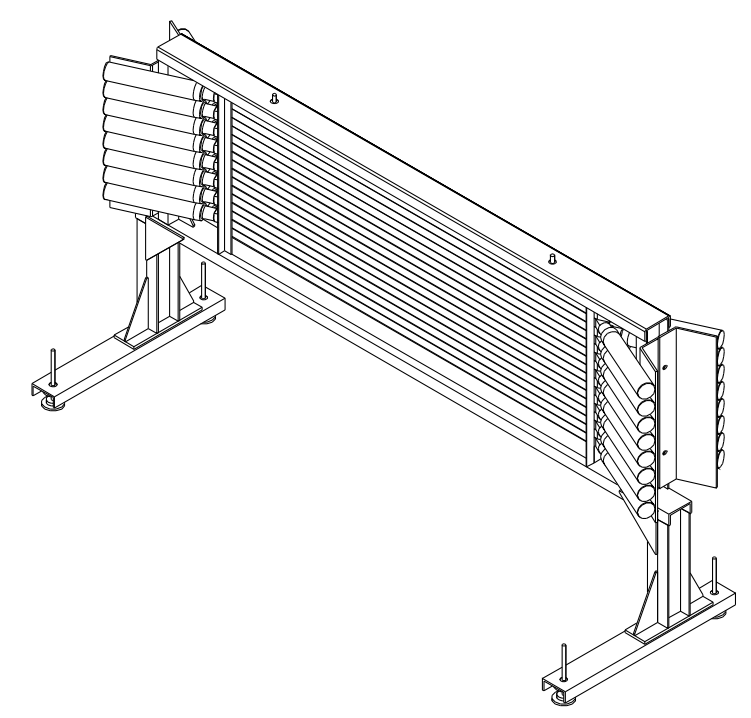

Fig. 24. General layout of the horizontal hodoscopes.

\section{The Horizontal Hodoscopes}

The horizontal hodoscope system $(\mathrm{HH})$ is also separated into two arms, each covering an area of $40 \times 130 \mathrm{~cm}^{2}$. The HH participates, together with the vertical hodoscope, to the definition of the first level trigger. Its response is used to apply a coplanarity criterion to track pairs hitting both detector arms. This trigger requirement selects oppositely charged particles with relative vertical displacement, $\Delta y$, less than $7.5 \mathrm{~cm}$.

Each hodoscope consists of 16 horizontal extruded scintillating slabs of dimensions $130 \times 2.5 \mathrm{~cm}^{2}$, with a thickness of $2.5 \mathrm{~cm}$. Both ends of each slab are coupled to specially shaped light-guides as illustrated in Fig. 24.

Photoconversion is done by Philips XP2008 photomultipliers, equipped, as for the $\mathrm{VH}$, with a voltage divider allowing high counting rate capability. The front-end electronics system contains the same elements as those described for the vertical hodoscopes. The single hit detection efficiency of $\mathrm{HH}$ is greater than $96.6 \%$ on both arms, and the time resolution is 320 ps.

\section{The Cherenkov Counters}

This detector is essential for rejection of the main background of electronpositron pairs from photon conversion, Dalitz pairs, and to a minor extent from resonance decays. It is used in the first level $\pi^{+} \pi^{-}$main trigger and in the calibration trigger to select $e^{+} e^{-}$pairs. 
It is structured in two identical threshold Cherenkov counters [28], each covering one spectrometer arm (see Fig. 25).

The gas radiator is enclosed in a volume defined by the entrance and exit windows, with dimensions $143 \times 56 \mathrm{~cm}^{2}$ and $336 \times 96 \mathrm{~cm}^{2}$, respectively. The chosen radiator is $N_{2}$ at normal temperature and pressure $\left(\theta_{\breve{C}}=1.4^{\circ}\right)$ and the counter length is $285 \mathrm{~cm}$.

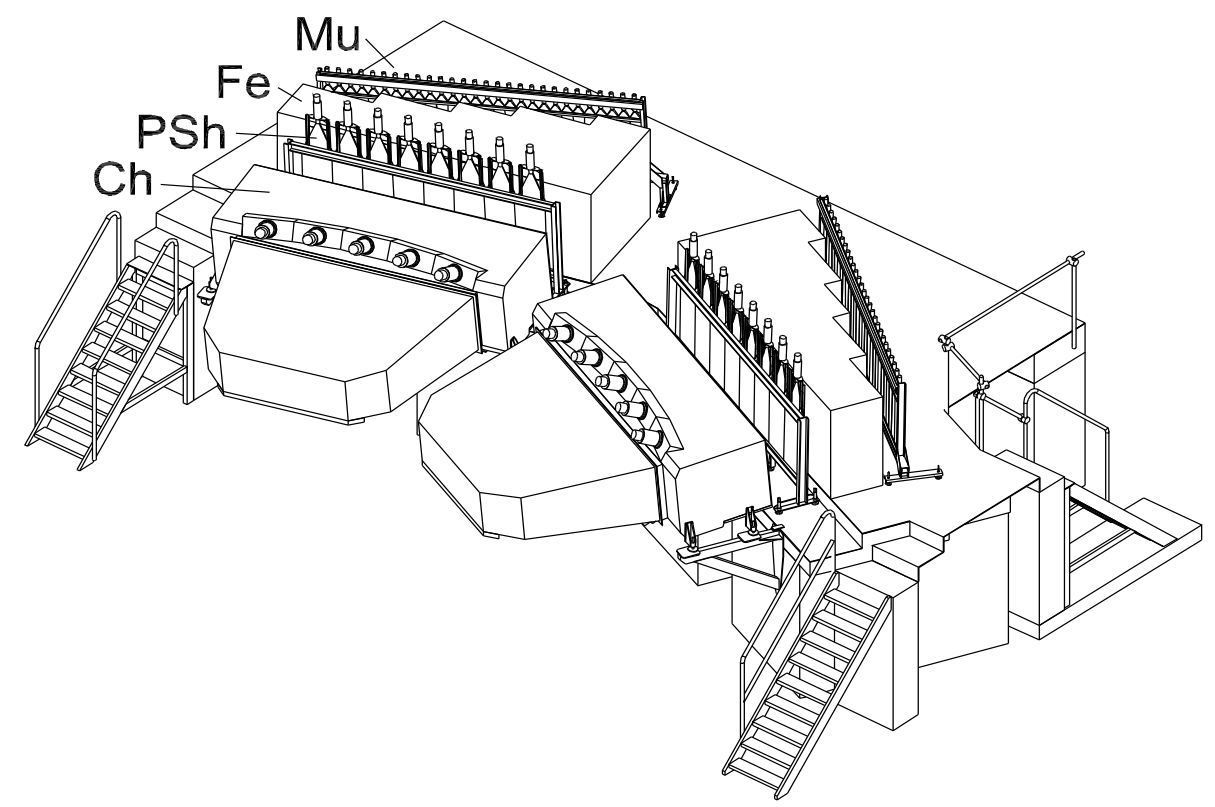

Fig. 25. The far end part of the DIRAC setup, comprising threshold Cherenkov counters $(\mathrm{Ch})$, preshower detector (PSh), iron absorber (Fe) and muon counters (Mu).

Each counter is equipped with 20 mirrors and 10 photomultipliers on two rows. Cherenkov light reflected by pairs of adjacent mirrors is focused onto the
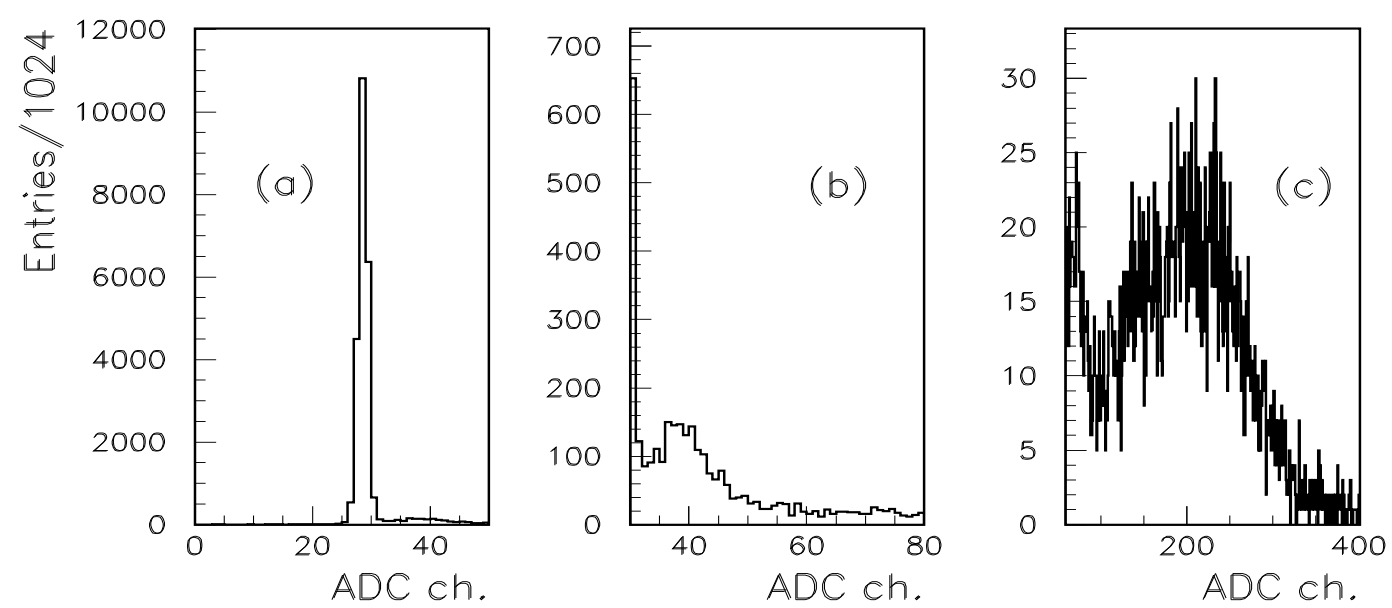

Fig. 26. ADC spectrum from one Cherenkov photomultiplier: (a) spectrum from pions (practically equal to the ADC pedestal distribution), (b) amplitude signal from single photoelectron, (c) spectrum from electrons. 

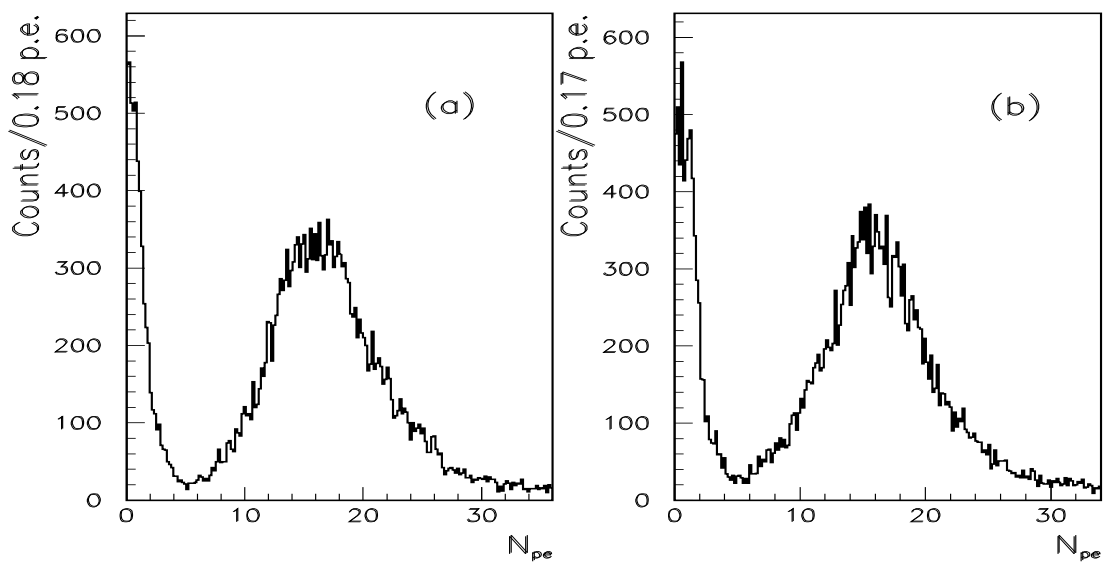

Fig. 27. Distribution of the number of photoelectrons detected from the (a) positive and (b) negative Cherenkov detector arms.

same photomultiplier (Hamamatsu R1587, with 130 mm UV-glass window). Mirrors and PM arrays are placed along circumferences with radii $630 \mathrm{~cm}$ and $570 \mathrm{~cm}$, respectively, centred at the magnet middle point. Mirrors are spherically deformed rectangles, with average dimensions $30 \times 35 \mathrm{~cm}^{2}$ and $6 \mathrm{~mm}$ thickness.

The analog signals from individual PM are fed into two custom-made summing modules, one per counter (10 channels input). The output of the summing module is a linear sum with fan-out of 2, plus the 10 input signals. At the module output the linear analog sum is attenuated by a factor of 3 with respect to individual channels. One analog sum is discriminated (LeCroy 4413 leading edge discriminators) and used for trigger purposes, whereas the individual PM signals and the other sum are fed, after a delay line, into LeCroy 4300B ADC units. Individual PM ADC spectra are used to perform overall amplitude alignment on the basis of maximum ratio between mean and r.m.s., in the PM voltage range $2400 \pm 200 \mathrm{~V}$.

A single photoelectron peak is clearly observed in all channels and it is shown in Fig. 26(b). The single photoelectron spectrum was used to cross-check the conversion factor from ADC counts to number of photoelectrons $\left(N_{p e}\right)$, which is obtained from the analysis of the summed amplitude spectra. Fig. 27 shows the latter as a function of $N_{p e}$ for the positive (a) and the negative (b) spectrometer arms. The mean values are $N_{p e}=16.2$ and $N_{p e}=16.4$, respectively. From these values we infer that both counters have an efficiency greater than $99.8 \%$ when operated at a threshold slightly less than 2 photoelectrons. The pion contamination above the detection threshold is estimated to be less than $1.5 \%$. Such contamination arises from pions with momenta above the Cherenkov threshold and from accidental coincidences occurring within the trigger time-window. 
Fig. 28. General picture of the spectrometer installed downstream the magnet, taken from a reference plane close to the Cherenkov counters, which can be appreciated in the bottom part of the picture. The magnet can be seen at the far end, and in between we see the vertical and horizontal hodoscope photomultiplier layout. The drift chamber system is installed behind it.

A general picture of the installed detectors described so far, looking into the magnet from a distance close to that of the Cherenkov counter, can be appreciated in Figure 28.

\section{The Preshower Detector}

The purpose of the Preshower detector (PSH) is two-fold: it provides additional electron/pion separation power in the off-line analysis and is used in the trigger generating logic as well.
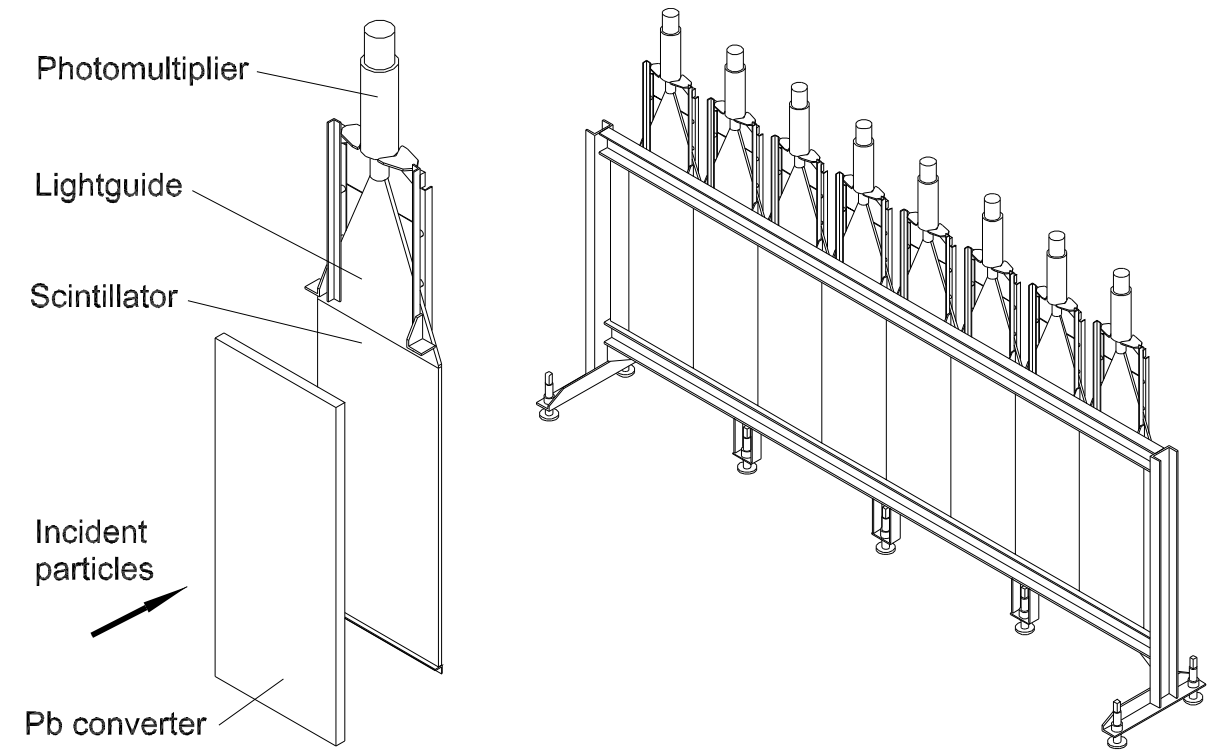

Fig. 29. PSH element and PSH array on one spectrometer arm.

The PSH is based on an array of lead converters followed by scintillation detectors [29]. Electrons (positrons) initiate in the converters electromagnetic showers which are sampled in the scintillation counters while pions behave mainly as minimum ionising particles. For trigger purposes, the signal has to be produced whether a pion or an electron cross a PSH counter.

The PSH consists of 16 detector elements placed symmetrically in two arms, as seen in Fig. 25. Each element has a Pb converter and a scintillation counter, Fig. 29. The converters of the two outermost elements of each arm (low mo- 
mentum region) are $10 \mathrm{~mm}$ thick, whereas the rest are $25 \mathrm{~mm}$ thick (around 2 and 5 units of radiation length, respectively). The scintillator used is BICRON type BC-408, with slab dimensions $35 \times 75 \mathrm{~cm}^{2}$ and $1 \mathrm{~cm}$ thickness. The scintillation light is transmitted to photomultipliers EMI 9954-B, placed at one end only, by $10 \mathrm{~mm}$ thick Plexiglas light-guides ending with Plexiglas cylinders to match with the PM photocathodes. Since the maximum particle flux on each PSH element is as high as $2 \mathrm{MHz}$, an additional booster power supply is used to feed the last PM dynodes.

The detector signals are linearly split into two branches, one used for trigger purposes and another for ADC analysis. In the former, a leading edge LeCroy 4416 discriminator is used with a threshold corresponding to efficient detection of minimum ionising particles. In the latter the signals are fed into $20 \mathrm{~dB}$ attenuators in order to fit the electron signals into the dynamical range of the LeCroy 4300B ADC.

The single arm detector efficiency is $99.5 \%$ for pions. Fig. 30 shows the pulseheight spectra from one element of the PSH for pions and electrons selected by the trigger system (this selection is based on the Cherenkov detector response).

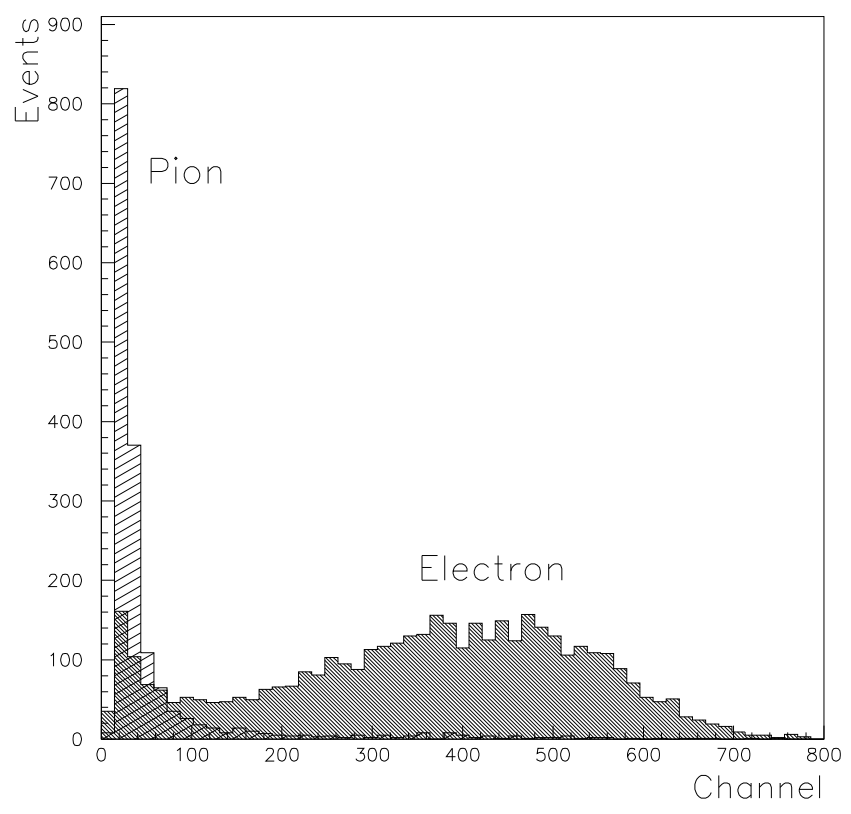

Fig. 30. Pulse-height spectra for pions and electrons in one element of PSH.

As seen from Fig. 30, the pion spectrum has a tail (originating from nuclear interaction of pions in the converter) extending to the electron amplitude region. The off-line study of the $e / \pi$ separation efficiency of the PSH showed that rejection of electrons reaches $85 \%$ with less than $5 \%$ losses of pions. As only a very small fraction of electrons escapes the on-line identification in the Cherenkov counters, the combined use of the Cherenkov and PSH detectors provides almost $100 \%$ electron rejection power at the off-line analysis stage. 


\section{Muon detector}

Admixture of muons in the $\pi \pi$ events can be a serious source of background. For this reason a muon detection system is implemented to provide efficient muon tagging. Muons come almost entirely from pion decays with a small admixture from other decays and direct $\mu^{+} \mu^{-}$pair production.

The muon detector consists of scintillation counters placed behind a thick iron absorber which almost entirely absorbs hadrons and related hadronic showers. This detector is placed at the downstream end of the DIRAC apparatus, few meters from the intense primary proton beam dump. As a result, the muon scintillation counters may undergo a high flux of background radiation from the beam dump area. This has required a special design of the counter arrays and electronics and has prevented from using muon information during on-line data reduction.

The counters are located behind iron absorber blocks with thickness ranging from 60 to $140 \mathrm{~cm}$ (see Fig. 25). The thickness is larger in the region close to the spectrometer symmetry axis, in order to compensate for the harder pion momentum spectrum. A double layer structure has been envisaged for the counters, each layer on each arm consisting of 28 counters with equal scintillating slabs of $75 \times 12 \mathrm{~cm}^{2}$ front area and $0.5 \mathrm{~cm}$ thickness. The muon detector data are read out only if simultaneous signals from a pair of corresponding counters in the two layers are detected. This essentially reduces the background counting rate induced by the neutron flux from the beam dump.

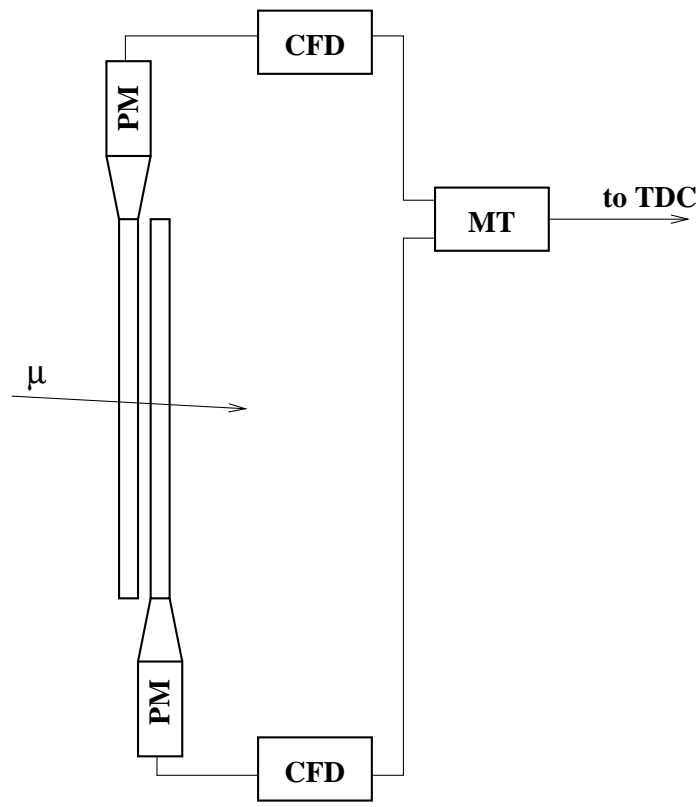

Fig. 31. Readout scheme of the muon detector element. 


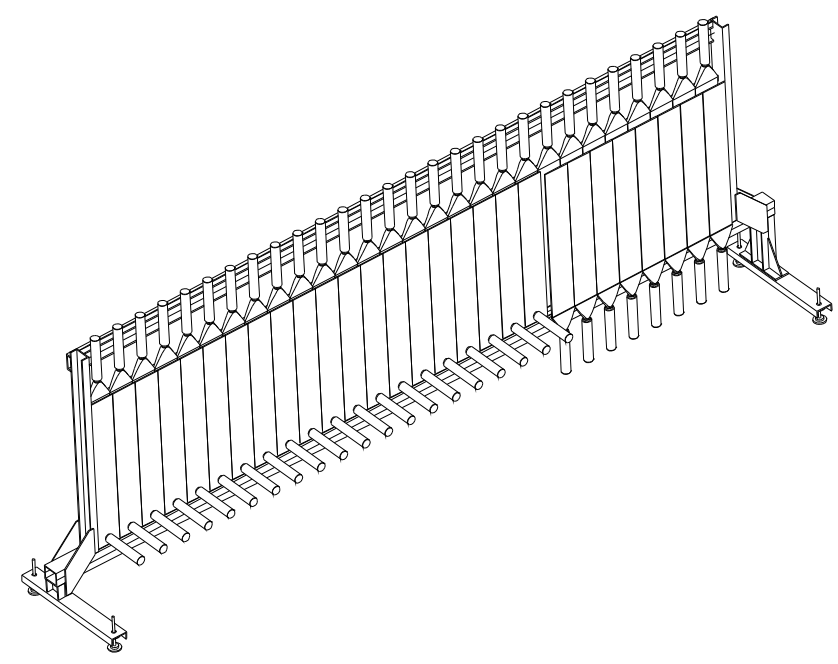

Fig. 32. Schematic layout of muon counters on their support structure, indicating light guides and photomultipliers.

A special readout architecture is realised to compromise between a cost-saving solution and the need to achieve a reasonably high time resolution. Scintillation light is detected by $25 \mathrm{~mm}$ diameter bialkaline FEU-85 photomultipliers placed at one scintillator end, in the two layers at opposite ends, as shown in Fig. 31. Signals from a pair of counters are fed into constant fraction discriminators (CFD) followed by meantimer (MT). CAEN modules C808 and C561 are used, respectively, for this purpose. In such a scheme the output signal is generated only if both counters are hit, and correct timing occurs only if the same particle crosses both counters.

Fish-tail light guides are used to couple the PM photocathodes to the scintillators, except when this is impeded by the presence of the concrete floor (see Fig. 32).

In this case (for 20 counters of the second layer in each arm), photomultipliers are directly coupled to the scintillating slabs, which are then made twice thicker to compensate for the loss of light yield.

In Fig. 33 the measured time difference between the signals of the muon detector and the vertical hodoscope is shown for an individual muon element of the positive arm. A global time resolution of $1.3 \mathrm{~ns}$ is observed, with a very small background level.

The way to suppress muons at the trigger level would be to include the muon counter signals into the anti-coincidence trigger logic. Because of the high background load to the muon detector, one might dangerously suppress useful pion events if they happen to occur on-time with background signals in the muon detector. That is why the option to use off-line the muon detector information has been chosen. In the off-line analysis only the events with muon 


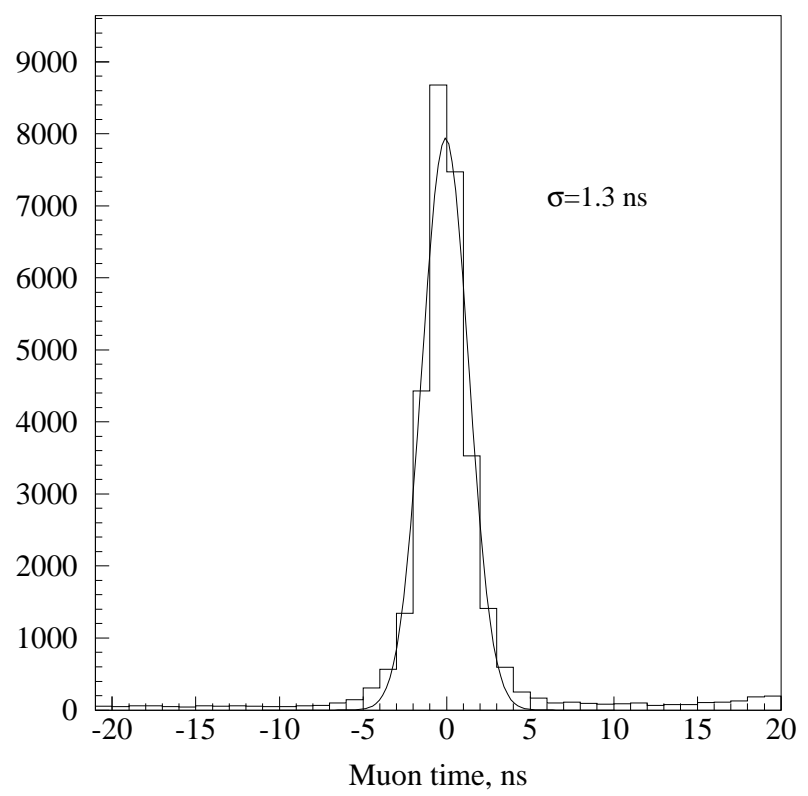

Fig. 33. Time difference between the signals of the muon detector and the vertical hodoscope.

counter hits correlated in time with those of other detectors are tagged as "muon" events and thus rejected [30]. From the analysis of experimental data we have inferred that the fraction of such events, containing at least one muon, is about $10 \%$ [31]. Such muon-events originate to a large extent $(\sim 80 \%)$ from $\pi^{ \pm}$-decays in the path between the DC and the muon counter systems (decays upstream the DC are mostly suppressed by the trigger system and thus contribute less to the collected event sample).

\section{Trigger system}

The trigger system was designed to provide a reduction of the event rate to a level acceptable to the data acquisition system which is around 2000 events/spill. Pion pairs are produced in the target mainly in a free state with a wide distribution over their relative momentum $Q$, whereas atomic pairs from $A_{2 \pi}$ disintegration have very low $Q$, typically below $3 \mathrm{MeV} / c$. The online data selection rejects events with pion pairs having $Q_{L}>30 \mathrm{MeV} / c$ or $Q_{x}>3 \mathrm{MeV} / c$ or $Q_{y}>10 \mathrm{MeV} / c$, keeping at the same time high efficiency for detection of pairs with $Q$ components below these values, $\left(Q_{L}, Q_{x}\right.$ and $Q_{y}$ are longitudinal and transversal components of the relative momentum, respectively.) 
A multilevel trigger is used in DIRAC [32]. It comprises a simple and fast first level trigger and higher level trigger processors which apply selection criteria to different components of the relative momentum of pion pairs.

Due to the requirements of the data analysis procedure, the on-line selection of only time correlated (prompt) pion pairs, originating from a single protontarget interaction and detected simultaneously by both spectrometer arms, is not enough. In addition, a large number of uncorrelated, accidental, pion pairs is also necessary. These accidental pairs are used in the off-line analysis to describe the relative momentum distribution of free (non-atomic) pion pairs without Coulomb interaction in the final state. Therefore, the trigger system should apply very similar selection criteria to prompt and accidental events, within a preselected coincidence time window centred around the peak of prompt events. The statistical error of the $A_{2 \pi}$ lifetime measurement depends on the number of both prompt and accidental detected pairs. In standard experimental conditions, the optimal ratio of prompt to accidental events is obtained using a 40 ns wide coincidence time window between the times measured in the left (VH1) and right (VH2) vertical hodoscopes.

Since 1999, when the experiment has started, the trigger architecture was upgraded several times to achieve a larger reduction of the background event rate (prompt and accidental pairs with large values of $Q$ ). In the present article we briefly describe the most recent version ${ }^{2}$.

A block diagram of the trigger architecture is presented in Fig. 34. The first level trigger $\mathrm{T} 1$ starts digitisation of the detector signals in the data acquisition (DAQ) modules (ADC, TDC, etc.). At the next level the neural network trigger DNA/RNA (DIRAC Neural Atomic and Revised Neural Atomic trigger) rejects the events with high $Q$ values. At the last stage, a powerful drift chamber trigger processor T4 imposes additional constraints to the relative momentum and takes the final decision to accept or to reject the event.

In addition to the main trigger designed to detect pionic atoms, several calibration triggers are run in parallel. They are applied to DAQ directly, without suppression by higher level trigger stages but with appropriate prescaling factors.

2 Before 2001 the trigger system included T2 and T3 stages following the first level trigger (T1). T2 selected particle pairs with a small $\Delta x$ distance in the upstream spectrometer region (for rejection of high $Q_{x}$ ) using the data from SFD and IH. T3 analysed hit patterns of the upstream $\mathrm{IH}$ and downstream VH detectors imposing selection criteria to $Q_{L}$ values. A detailed description of T2 and T3 is given in [32] and [33]. With the implementation of a new architecture of the trigger system these trigger stages were removed. 


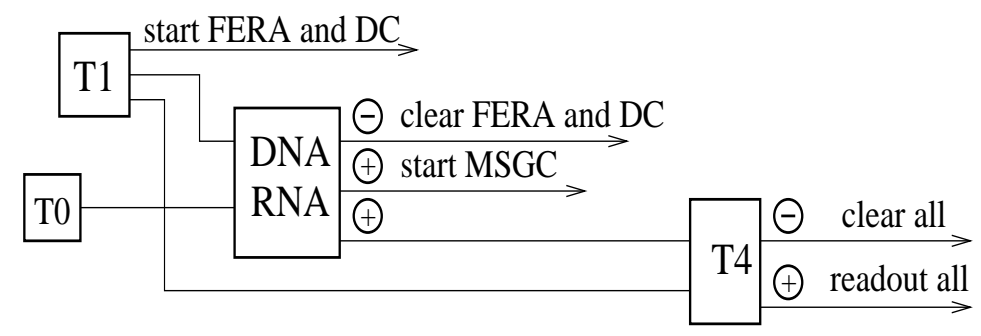

$\odot$ and $\Theta$ denote positive and negative decisions of the corresponding trigger level

Fig. 34. General block diagram of the DIRAC multilevel trigger.

\subsection{First level trigger (T1)}

The first level trigger (a detailed description is given in [34]) fulfils the following tasks:

- Selects events with signals in both detector arms downstream the magnet.

- Classifies the particle in each arm as $\pi^{ \pm}$or $e^{ \pm}$depending on the presence of the Cherenkov counter signal. Protons, kaons and muons are equally included in the "pion" class, their identification is performed in the off-line analysis.

- Arranges the coincidences between the signals detected in the two arms. The width of the coincidence time window defines the ratio between yields of prompt and accidental events in the collected data.

- Applies a coplanarity criterion to particle pairs: the difference between the hit slab numbers in the horizontal hodoscopes in the two arms (HH1 and $\mathrm{HH} 2$ ) should be $\leq 2$. This criterion forces a selection on the $Q_{y}$ component of the relative momentum and provides a rate reduction by a factor of 2 .

- Selects in parallel events from several physics processes needed for the setup calibration: $e^{+} e^{-}$pairs, $\Lambda \rightarrow p+\pi^{-}$decays, $K^{ \pm}$decays to three charged pions.

The physics and calibration trigger signals pass through the mask register and, after proper prescaling, are combined with an OR function. Any trigger type can be enabled or disabled by proper programming of the mask register. Independent prescaling of each sub-trigger channel allows to adjust their relative rate with respect to the rate of the main trigger. A specific trigger mark is recorded for every event to allow sorting the data by trigger type during off-line analysis and on-line monitoring.

All T1 modules are ECL line programmable multichannel CAMAC units. Most of them are commercial modules, except for the dedicated coplanarity processor which has been custom-developed at JINR. Meantimer units are used in 
all $\mathrm{VH}$ and $\mathrm{HH}$ channels to remove the dependence of the time measurement on the hit location, thus reducing the total trigger time jitter.

\subsection{Neural network trigger (DNA/RNA)}

The DNA/RNA trigger [35] is a processing system using a neural network algorithm. Its hardware is based on the custom-built version of the neural trigger used in the CPLEAR experiment [36].

DNA/RNA receives (see Fig. 35) the hit patterns from the vertical hodoscopes VH1, VH2 and the X-planes of the upstream detectors: the ionisation hodoscope (IH) and the scintillating fibre detector (SFD). For low $Q$ events the hits in these detectors are correlated.

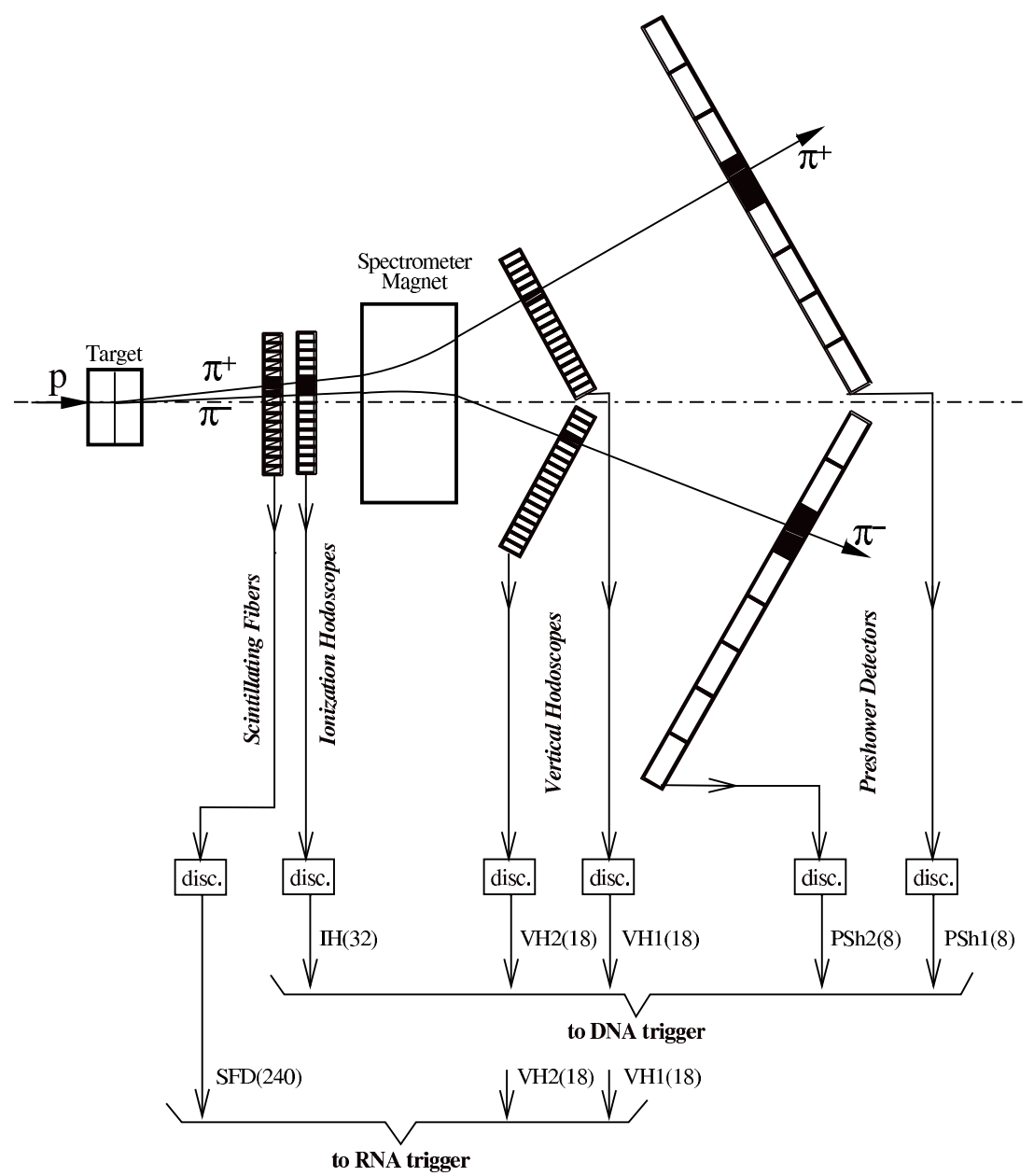

Fig. 35. DIRAC detectors used for the neural network triggers DNA and RNA. Numbers of signal channels from each detector are given in parentheses. 
The neural network was trained to select particle pairs with low relative momenta: $Q_{x}<3 \mathrm{MeV} / c, Q_{y}<10 \mathrm{MeV} / c$ and $Q_{L}<30 \mathrm{MeV} / c$. The events which do not satisfy any of those conditions are considered "bad" and rejected.

The DNA/RNA logic is started by a fast pretrigger, T0, and in 250 ns evaluates an event. Rate reduction by a factor of 2 with respect to $\mathrm{T} 1$ is achieved with DNA/RNA.

\subsection{Drift chamber processor (T4)}

$\mathrm{T} 4$ is the final trigger stage. T4 processor reconstructs straight tracks in the $\mathrm{X}$-projection of the drift chambers and analyses them to determine the value of the relative momentum (the algorithm is described in [32]).

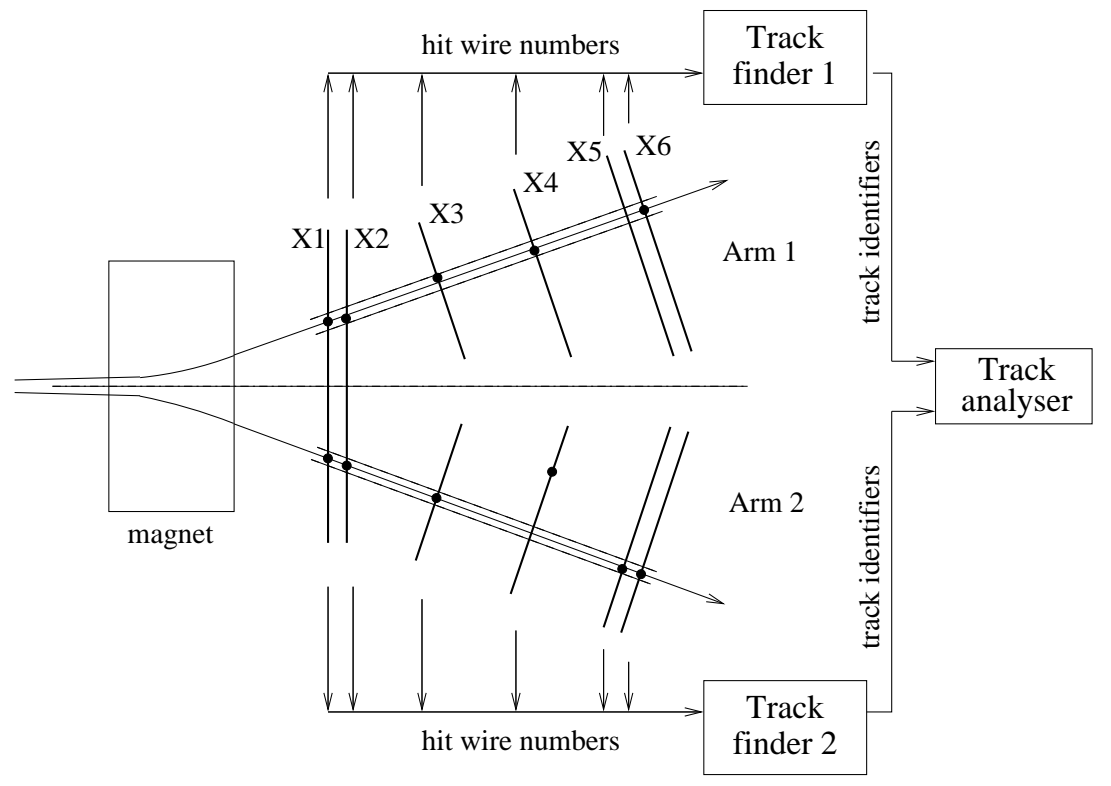

Fig. 36. T4 operation block diagram. Only the drift chamber X-planes involved in T4 are shown.

The block diagram of the T4 operation is shown in Fig. 36. The drift chamber processor includes two stages: the track finder and the track analyser. The track finder (an identical processor is used for each arm) receives the numbers of the hit wires from all drift chamber X-planes. Drift time values are not used in the T4 logic. A unique number, "track identifier", which contains the encoded numbers of the hit wires, is associated to the found track. 
If tracks are found in both arms, the track analyser continues the event evaluation. The track analyser receives the track identifiers from both arms and compares them with the content of a look-up memory table which contains all possible combinations of track identifiers for pion pairs with $Q_{L}<30 \mathrm{MeV} / c$ and $Q_{x}<3 \mathrm{MeV} / c$. These "allowed" combinations are obtained from a dedicated simulation using the precise geometry of the setup. If a relevant combination is found, the T4 processor generates a positive decision signal which starts the data transfer to the VME buffer memories. Otherwise, the Clear and Reset signals are applied to the DAQ and trigger systems.

The T4 decision time depends on the complexity of the event and is around $3.5 \mu \mathrm{s}$ on average. The rejection factor of $\mathrm{T} 4$ is around 5 with respect to the T1 rate and around 2.5 with respect to DNA/RNA.

The whole trigger system is fully computer controlled: no hardware intervention is needed in order to modify the trigger configuration. With all selection stages enabled the event rate at the typical experimental conditions is around 700 per spill, that is well below the limits of the DAQ rate capability.

\section{Data acquisition system}

The architecture of hardware and software parts of the data acquisition system takes into account the time structure of the proton beam. The machine supercycle of the CERN PS accelerator has 15-20 s duration. Within this period DIRAC receives protons in spills of 400-450 ms width, from 1 to 5 spills per super-cycle. The interval between consecutive spills can be as short as $1 \mathrm{~s}$.

During the accelerator burst the data from all detectors are read out into VME buffer memories (commercial and dedicated electronic modules) without any software intervention. The data transfer to VME processor boards, event building, data transfer to the main host computer and other relatively slow operations are performed during the pause between bursts. This provides the maximum operation rate of DAQ. The information comes from 2048 channels of microstrip gas chambers, 800 channels of scintillating fibre detector, 2016 channels of drift chambers and 224 channels of other scintillation and Cherenkov detectors. For every channel the time or amplitude information is recorded or both. In addition to a main readout mode, the readout of scalers at the end of every spill is arranged via a CAMAC bus. The counting rates of all the detectors, trigger rates at different trigger levels and for different sub-trigger modes are recorded together with the value of the beam intensity provided by the PS complex. 


\subsection{DAQ hardware}

The data readout [37] is arranged with 12 readout branches: 4 branches for GEM/MSGC, 3 branches for DC and 5 FERA [38] branches for all other detectors. In FERA and DC branches the VME modules CES HSM 1870 and LeCroy 1190 are used as buffer memories. In GEM/MSGC branches the buffer memories are incorporated into dedicated VME modules [39]. FERA branches include different FERA compatible LeCroy modules: ADC 4300B, multi-hit TDC 3377, universal logic modules 2366 configured in this application like FERA registers and scalers. Peculiarities of FERA readout in DIRAC, such as multi-gate and Fast Clear operation, are described in [40].

The logic of readout is the following. The first level trigger T1 starts digitisation in ADC and TDC of FERA and DC branches (see Fig. 34 in Section "Trigger system"). Readout is inhibited unless a positive decision of the highest level trigger T4 is received. If the T4 processor decides positively, the Inhibit Readout status is released and the converted event data are transferred to buffer memories. If the decision of DNA/RNA or T4 is negative, then a Fast Clear signal is generated [40] which discards the data in all FERA modules and DC registers.

In contrast to FERA and DC subsystems, the processing of GEM/MSGC data is started by the next level DNA/RNA trigger. This reduces the dead time introduced by the Clear process which in GEM/MSGC electronics takes longer. Thus, the negative decisions of T4 only lead to clearing of the GEM/MSGC data. If no Clear signal is received, the converted data are transferred to buffer memories.

The readout of the whole event takes $45 \mu$ s and is defined by a fixed acquisition time of GEM/MSGC which exceeds the readout time in other branches.

\subsection{DAQ software}

The main part of the DAQ software [41] is running on two VME processor boards and on the main DAQ host. PowerPC-based VME processor boards control the trigger and FERA readout electronics via two CAMAC branch drivers, operate VME modules, read data from buffer memories and transfer them to the main DAQ host. The main DAQ host performs event building, records the built data and distributes them to other computers for on-line monitoring and analysis. 
The DAQ software is written in C programming language and is running under UNIX-like operating systems: Lynx-OS (VME processors) and LINUX (main DAQ host).

The schematic layout of the data acquisition processes is presented in Fig. 37. The basic processes running on VME and main DAQ hosts and on one of the monitoring computers are shown. Only one VME processor is shown for simplicity.

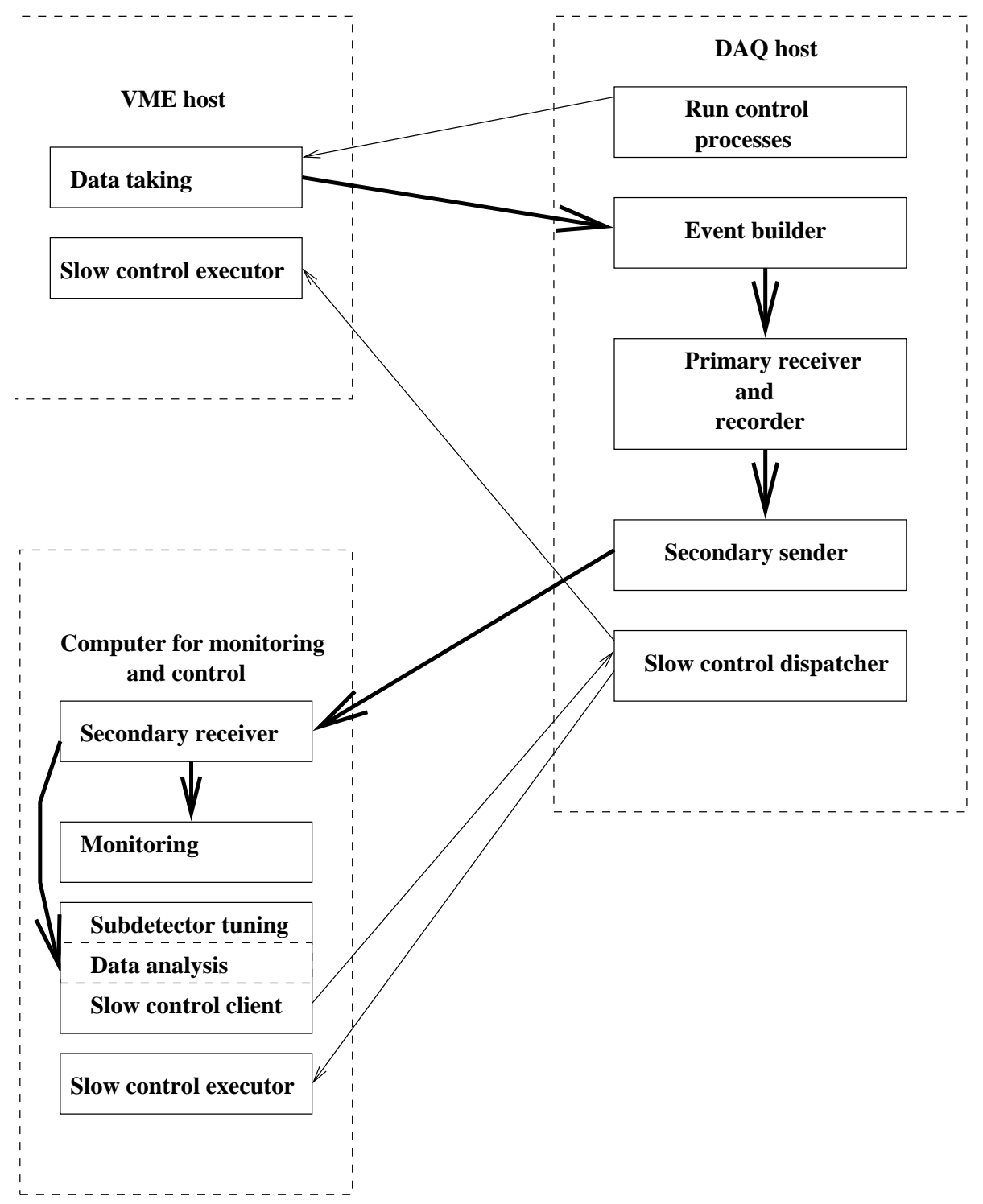

Fig. 37. Layout of the DAQ architecture.

The DAQ software can be divided into more or less independent groups. The first one is a set of programs for data readout (runs on VME boards) and event building (runs on the main DAQ host). During the event building the data are checked for consistency using the headers and the serial numbers of sub-events provided by the detector readout electronics. The serial numbers 
allow to check that all buffer memories contain the same number of sub-events and that different sub-events correspond to each other.

The second group consists of programs for data distribution over the network for on-line monitoring. The group includes a primary data receiver and secondary data senders/receivers. The primary receiver is the only program which receives data directly from the event builder and is critical for the data acquisition: other processes of this group may either be or not be running and may be started or stopped at any time.

The third group of programs allows to control the run status via a graphical user interface: to set the run parameters, to select the trigger type, to change the run status (start, stop, suspend etc.). This group includes also a Run Display program which delivers information about the current run conditions.

The fourth group is a set of slow-control processes providing a uniform access to hardware from any host participating in DAQ. The tasks of these programs are, for example, high voltage control, VME and CAMAC module initialisation and so on.

The whole DAQ system is able to accept data from five consecutive bursts in one accelerator super-cycle with up to 2 MBytes per burst (this corresponds to 2000 triggers per burst) and to distribute events to all hosts participating in on-line data processing. The limitation comes from the capacity of the VME buffer memories.

\subsection{On-line monitoring}

An on-line monitoring program receives the data distributed by the main DAQ process. The program fulfils accumulation of several hundred histograms, including all raw signal spectra from any detector as well as spectra obtained after a fast preliminary data handling. The software is written in the frame of ROOT software package.

The program delivers the following information:

— Time and amplitude distributions for every counter.

- Hit and multiplicity distributions.

- Correlation plots for hits in different detectors.

- Beam profile on the experimental target, beam spill time structure.

- Some specific data for selected detectors (cluster size for GEM/MSGC etc.).

- Event rates for different trigger modes running in parallel and their timing. 
- Information about the data taking process as a whole: burst data volume, event volume, time of event in burst etc.

Proper setting of the corresponding program parameter allows selection of the trigger type for which all the above operations should be fulfilled. For example, the histograms can be obtained separately for $e^{+} e^{-}, \pi^{+} \pi^{-}, \Lambda$ and any other trigger or for all accepted triggers.

Apart from the common program, there are dedicated monitoring programs developed for more detailed control of individual detectors.

\section{Setup performances}

The experimental setup is designed to provide high efficiency for detection of $\pi^{+} \pi^{-}$pairs with small opening angle in the laboratory frame $(\theta<3 \mathrm{mrad})$ and small relative momentum in the center of mass frame $(Q<3 \mathrm{MeV} / c)$. Detector resolution and multiple Coulomb scattering in the target and setup elements affect the measurement accuracy.

Detector resolution leads to a linear increase of the relative error $\sigma_{p} / p$ with increasing momentum value, whereas multiple scattering contributes a constant term to the momentum uncertainty. With an experimental space resolution of $90 \mu \mathrm{m}$ in the DC system and $50 \mu \mathrm{m}$ in the GEM/MSGC detectors, the uncertainty on the coordinate and momentum measurements are mainly determined by the description of the multiple scattering errors.

Both momentum and the opening angle measurement accuracies affect the precision on the measurement of the relative momentum $Q$ of the pion pair. The momentum accuracy $\sigma_{p} / p$ is $0.3 \%$, at $p_{\pi}=p_{A_{2 \pi}} / 2=2 \mathrm{GeV} / c$, almost independent on momentum.

The kinematics of $\pi^{+} \pi^{-}$pairs originating from the ionisation of $A_{2 \pi}$ atoms in the target requires ability on two-track separation in the upstream detector region better than $1.5 \mathrm{~mm}$ in most of the cases. The experimental double track resolution is limited by the granularity and signal clustering of the upstream detectors and with current algorithms is about $0.4 \mathrm{~mm}$. Thus, the accuracy on the measurement of the longitudinal $\left(Q_{L}\right)$ and transversal components $\left(Q_{x}\right.$ and $\left.Q_{y}\right)$ of $Q$ is $0.6 \mathrm{MeV} / c\left(Q_{L}\right)$ and $0.4 \mathrm{MeV} / c\left(Q_{x}\right.$ and $\left.Q_{y}\right)$. These errors arise from the estimated contribution of multiple scattering in the detectors and setup elements, according to our present knowledge of the experimental data. In fact, the effect of multiple scattering in the foil target increases the error in $Q_{x}$ and $Q_{y}$ to up $1 \mathrm{MeV} / c$. 


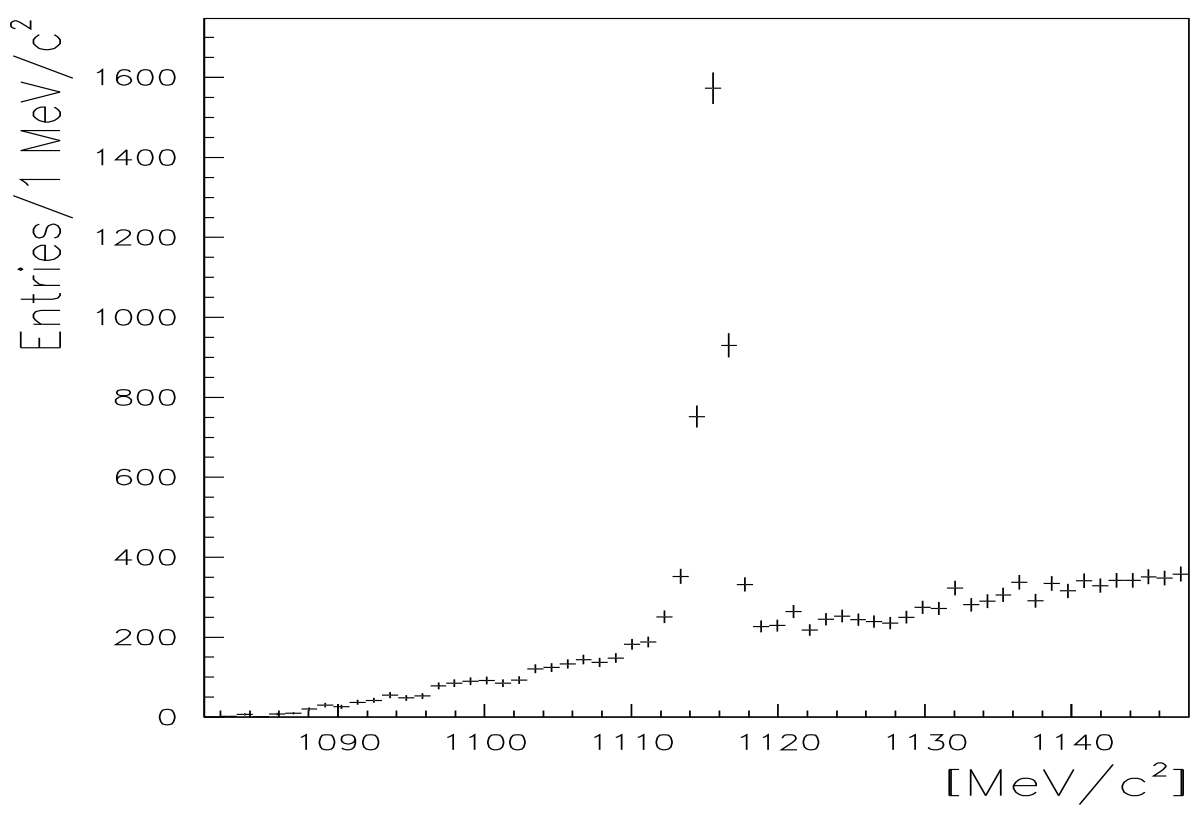

Fig. 38. Invariant mass distribution of $p \pi^{-}$pairs. Events were selected from a sample of $\Lambda$ calibration data.

The calibration of the energy scale and the experimental determination of the setup resolution are performed by monitoring the position and the width of the $\Lambda$ detected by the apparatus. The reconstructed $p \pi^{-}$invariant mass obtained from calibration data is shown in Fig. 38. The $\Lambda$ is clearly seen above a small background. A gaussian plus a polynomial fit to the data gives $M_{\Lambda}=1115.67 \mathrm{MeV} / c^{2}$ and $\sigma_{\Lambda}=0.43 \mathrm{MeV} / c^{2}$. Calibration using $\Lambda \rightarrow p \pi^{-}$ decays is used in addition to control the precision of the setup alignment. Any misalignment of the tracking system in one arm relative to the other arm would result in asymmetrical errors on the reconstructed momenta.
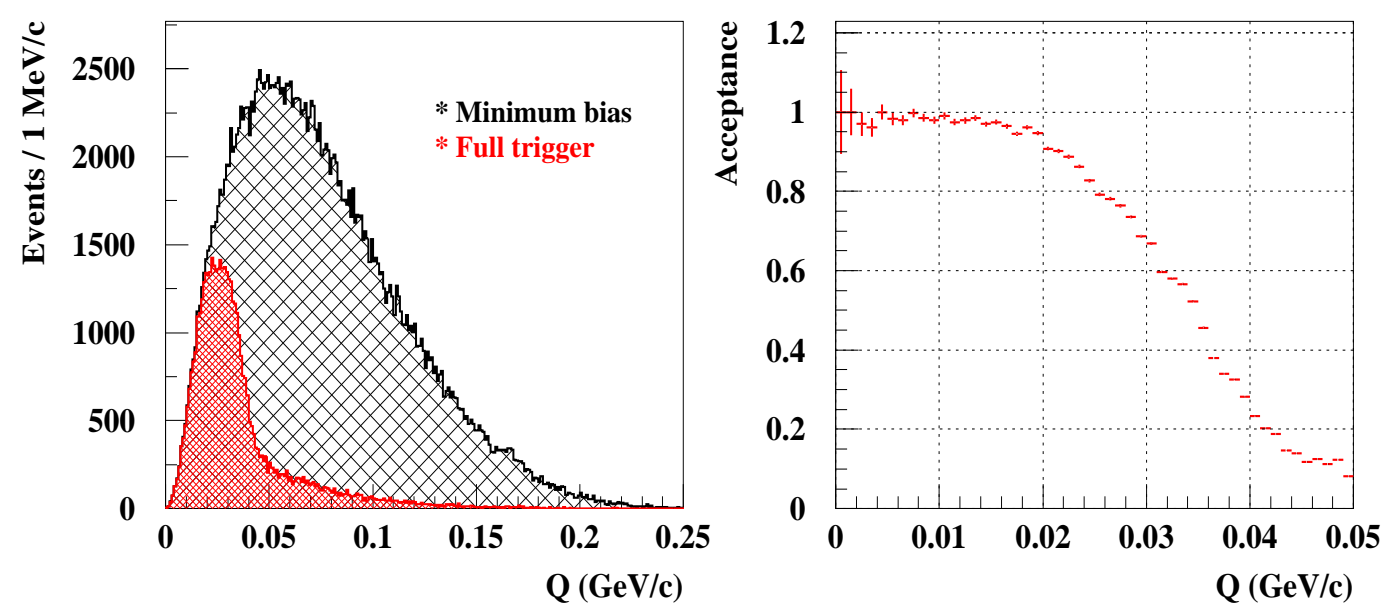

Fig. 39. Left: Distribution of $Q$ for accepted $\pi^{+} \pi^{-}$pairs after the full DIRAC trigger system, and for minimum bias pairs. Right: trigger acceptance, determined as the ratio between the previous two distributions. 

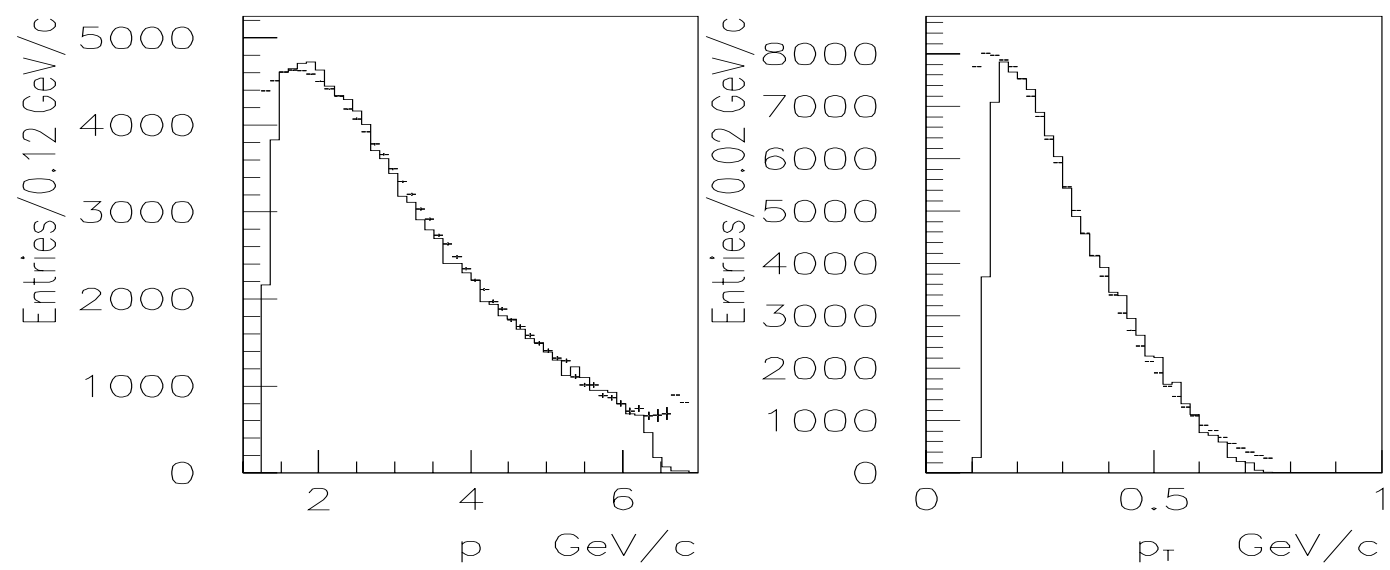

Fig. 40. Experimental $p$ and $p_{T}$ distributions of $\pi^{-}$corrected for apparatus acceptance (histogram) with superimposed the results of the parameterisation [42][43].

The accuracy of the time measurement is obtained from the analysis of the distribution of the $e^{+} e^{-}$time difference at the vertical hodoscopes as explained in that section (see Fig. 22).

The performance of the trigger system as a whole in selecting low- $\mathrm{Q}$ events is illustrated in Fig 39, where the magnitude of relative momentum of pion pairs $Q$ is shown (in their center-of-mass frame), after DNA/RNA and T4 trigger selection (see trigger section). Trigger efficiency as function of $Q$ is flat in the low-Q region, as illustrated in Fig. 39. This is considered an important figure of merit of the spectrometer, for a precision study of the $\pi^{+} \pi^{-}$Coulomb interaction.

Pions from ionisation of $A_{2 \pi}$ entering the apparatus have momenta below $4 \mathrm{GeV} / c$. The apparatus momentum acceptance for time-correlated pairs is flat for pions with momenta between $1.6 \mathrm{GeV} / c$ and $3 \mathrm{GeV} / c$, and it decreases for higher momenta.

For the sake of completeness we also show the $p$ and $p_{T}$ distributions for a single $\pi^{-}$in Fig. 40, with superimposed a parameterisation of the inclusive yield based on the analytic representation [42], adapted to DIRAC center-ofmass energy $(\sqrt{s}=6.84 \mathrm{GeV})[43]$.

\section{Acknowledgements}

We would like to acknowledge the financial support received by the institutions in the DIRAC collaboration for the construction of the spectrometer, in particular by the following funding agencies: the Swiss National Science Foundation, the Ministerio de Ciencia y Tecnologia (Spain), under projects 
AEN96-1671 and AEN99-0488, the PGIDT of Xunta de Galicia (Spain), the Istituto Nazionale di Fisica Nucleare (Italy), the Ministery of Industry, Science and Technologies of the Russian Federation and the Russian Foundation for Basic Research (Russia), under project 01-02-17756, the IN2P3 (France), the Greek General Secretariat of Research and Technology (Greece), the University of Ioannina Research Committee (Greece), the Grant Agency of the Czech Republic, grant No. 202/01/0779, and the Japan Society for the Promotion of Science (JSPS), Grant-in-Aid for Scientific Research No. 07454056, 08044098, 09640376, 11440082, 11694099, 12440069 and 14340079.

We wish to thank the CERN Directorate for the continuos encouragement and support to DIRAC. We also thank the personell of the CERN PS Division for their essential contribution to the experiment. We warmly acknowledge the help of J. Bosser, A. Braem, M. Bragadireanu, N. Chritin, L. Danloy, M. Doser, B. Dulach, L. Durieu, O. Ferrando, W. Flegel, A. Froton, J. Ch. Gayde, P. A. Giudicci, M. Hauschild, J. Y. Hemery, M.G. Iovanozzi, G. Martini, G. Molinari, J.M. Nonglaton, V. Prieto, J.P. Riunaud, T. Ruf, D. Simon, R. Steerenberg, Ch. Steinbach, J.W.N. Tuyn, M. Zahnd and M. Zanolli.

We are also grateful to the Directorate of Joint Institute for Nuclear Research, for its support to the experiment.

Finally would like to thank our secretary C. Moine for the care she has put in solving our every-day problems.

\section{References}

[1] J. Uretsky and J. Palfrey, Phys. Rev. 121 (1961) 1798.

S.M. Bilenky et al. Yad. Fiz. 10 (1969) 812.

J. Gasser, V.E. Lyubovitskij, A. Rusetsky, A. Gall, Phys. Rev. D64 016008 (2001).

[2] G. Colangelo, J. Gasser, H. Leutwyler, Nucl. Phys. B603 (2001) 125.

[3] B. Adeva, et al., "Lifetime measurement of $\pi^{+} \pi^{-}$atoms to test low energy QCD predictions", CERN/SPSLC 95-1, SPSLC/P 284, Geneva 1995.

[4] L. Afanasyev et al. Phys. Lett. 308 B (1993) 200.

[5] L.L. Nemenov, Yad. Fiz. 41 (1985) 980.

[6] A. Kuptsov, V. Yazkov, "Dirac experimental setup II - Layout and characteristics of the detectors", DIRAC note 1996-23, CERN, 1996.

[7] A. Kuptsov, "The DIRAC setup (drawings)", DIRAC note 1997-15, CERN, 1997. 
[8] K. Brown, D. Carey, C. Iselin, F. Rothacker. "TRANSPORT: a computer program for designing charged-particle beam transport systems", CERN-80-04, Geneva, CERN, 1980.

[9] J. Bosser, G. Molinari, J-M. Nonglaton, V. Prieto, R. Steerenberg, "Test of a dispersion sweep correction system using a centroid in the DIRAC beam line", CERN/PS 2002-041 (OP), Geneva, CERN, 2002.

[10] I.A. Kurochkin, "Preliminary calculations of neutron background in the DIRAC experiment", DIRAC note 96-7, CERN, 1996.

[11] F. Sauli, NIM A386 (1997) 531.

[12] "Development of a Micro Strip Detector for the DIRAC Experiment", Teresa Núñez, Doctoral Thesis, University of Santiago de Compostela, 1999.

[13] A. Oed, NIM A263 (1988) 351.

[14] F. Gómez et al., Nucl. Inst. and Meth. A384 (1997) 351.

[15] B. Adeva et al., "Performance of the GEM/MSGC detector in the DIRAC Experiment", Nucl. Inst. and Meth. A, in preparation.

[16] Manufacturing technology by the CERN EST DEM group.

[17] "Development and Construction of the Readout Electronics of Micro Strip Detectors", Pablo Vázquez, Doctoral Thesis, University of Santiago de Compostela, 2000.

[18] R. Horisberger and D. Pitzl, Nucl. Inst. and Meth. A326 (1993) 92.

[19] V. Agoritsas et al., Nucl. Instr. and Meth., A411 (1998) 17-30.

[20] V. Agoritsas et al., "Ultrafast readout of scintillating fibres using upgraded position-sensitive photomultipliers" , DRDC Proposal, DRDC/P-25, CERN, 1991.

[21] M. Ferro-Luzzi et al., Notre Dame 1997, "Scintillating fibre detectors", AIP Conf.Proc. Vol.450, p.278-285, New York, USA.

[22] A. Gorin et al., Nucl. Instr. and Meth., A452 (2000) 280-288.

[23] V. Brekhovskikh et al., "New Ionisation Hodoscope: design and characteristics", DIRAC note 2002-09, CERN, 2002.

[24] C. Detraz, D. Drijard, M. Ferro-luzzi and V. Komarov, "The ionisation hodoscope: performance and characteristics of the first module", DIRAC Internal Note 1997-09.

[25] Millipore paper type HAWP pore size 0.45 made by MILLIPORE Corp., Bedford, Ma 01730 USA.

[26] L. Afanasyev, V. Karpukhin, NIM A492 (2002) 351.

[27] B. Adeva et al., Nucl. Inst. and Meth. A491 (2002) 41. 
[28] M. Bragadireanu et al., NIM 426 (1999) 254.

[29] M. Pentia, Gh. Caragheorgheopol, M. Ciobanu, D. Pop and C. Rusu, "Preshower detector commissioning along with DIRAC set-up", DIRAC note 99-03.

[30] V. Brekhovskikh, M.V. Gallas, "Muon identification in DIRAC experiment", DIRAC Note 2001-02.

[31] A. Lanaro, "Features of $\mu$-candidate events", DIRAC Note 2002-02.

[32] L. Afanasyev et al., NIM A491 (2002) 376.

[33] M. Gallas, NIM A482 (2002) 222.

[34] L. Afanasyev, M. Gallas, V. Karpukhin and A. Kulikov, NIM A479 (2002) 407.

[35] P. Kokkas, M. Steinacher, L. Tauscher and S. Vlachos, NIM A471 (2001) 358.

[36] F.R. Leimgruber et al., NIM A365 (1995) 198.

[37] V. Karpukhin, A. Kulikov, V. Olshevsky, S. Trusov. "Readout logic and its hardware implementation in the DIRAC experiment." Preprint JINR E10-20032, Dubna, 2003. Submitted to NIM.

[38] FERA - Fast Encoding and Readout ADC System Possibilities. Application Note AN-4004A, LeCroy Corporation.

[39] F. Gomez, P. Vazquez, "MSGC/GEM Detector Electronics", DIRAC Internal Note 00-01, CERN, 2000.

[40] V. Karpukhin, A. Kulikov, "Multigate and Fast Clear Logic in FERA Readout", Preprint JINR E10-2001-2, Dubna, 2001.

[41] V.G. Olshevsky, S.V. Trusov, NIM A469 (2001) 216.

[42] G.D. Badhwar, S.A. Stephens, R.L. Golden Phys Rev. D15, 820 (1977).

[43] A. Lanaro, "Parameterisation of correlated and accidental particle spectra", DIRAC Internal Note 01-01, CERN, 2001. 